\title{
Half-sandwich silane $\sigma$-complexes of ruthenium supported by NHC carbene
}

\author{
Van Hung Mai, Ilia Korobkov, Georgii Nikonov
}

Chemistry Department, Brock University, Niagara Region, 1812 Sir Isaac Brock Way, St. Catharines, ON L2S 3A1, Canada. Tel.: +1 (905) 6885550, ext 3350, Fax: +1 (905) 6829020

\author{
Corresponding Author \\ * Georgii Nikonov, gnikonov@brocku.ca
}

\section{Supporting Information}

Table S1. Crystal and structure refinement data for $\mathbf{4 b}, \mathbf{4 d}, \mathbf{8}$, and 10

Figure S1. (a) ${ }^{1} \mathrm{H}$ NMR $\left(600 \mathrm{MHz}, 22^{\circ} \mathrm{C}, \mathrm{C}_{6} \mathrm{D}_{6}\right)$ of $\mathrm{Cp}(\mathrm{IPr}) \mathrm{RuCl}\left(\eta^{2}-\mathrm{HSiCl}_{3}\right)(4 \mathbf{a})$; (b) ${ }^{1} \mathrm{H} \mathrm{NMR}(600 \mathrm{MHz}$, $\left.-40{ }^{\circ} \mathrm{C}, \mathrm{C}_{6} \mathrm{D}_{5} \mathrm{CD}_{3}\right)$ of in situ generated $\mathrm{Cp}(\mathrm{IPr}) \mathrm{RuCl}\left(\eta^{2}-\mathrm{HSiCl}_{3}\right)(\mathbf{4 a})$

Figure S2. ${ }^{1} \mathrm{H}-{ }^{29} \mathrm{Si}$ INEPT NMR (119.2 MHz, $\left.-40{ }^{\circ} \mathrm{C}, \mathrm{C}_{6} \mathrm{D}_{5} \mathrm{CD}_{3}\right)$ of $\mathrm{Cp}(\mathrm{IPr}) \mathrm{RuCl}\left(\eta^{2}-\mathrm{HSiCl}_{3}\right)(\mathbf{4 a}) \quad \mathrm{S} 6$

Figure S3. ${ }^{1} \mathrm{H}-{ }^{29} \mathrm{Si}$ HSQC NMR (119.2 MHz, $\left.-40{ }^{\circ} \mathrm{C}, \mathrm{C}_{6} \mathrm{D}_{5} \mathrm{CD}_{3}\right)$ of $\mathrm{Cp}(\mathrm{IPr}) \mathrm{RuCl}\left(\eta^{2}-\mathrm{HSiCl}_{3}\right)(\mathbf{4 a}) \quad \mathrm{S} 6$

Figure S4. (a) ${ }^{1} \mathrm{H}$ NMR $\left(600 \mathrm{MHz}, 22^{\circ} \mathrm{C}\right.$, in $\left.\mathrm{C}_{6} \mathrm{D}_{6}\right)$ of $\mathrm{Cp}(\mathrm{IPr}) \mathrm{RuCl}\left(\eta^{2}-\mathrm{HSiCl}_{2} \mathrm{Me}\right)(\mathbf{4 b})$; (b) ${ }^{1} \mathrm{H} \mathrm{NMR}$ $\left(600 \mathrm{MHz},-40^{\circ} \mathrm{C}, \mathrm{C}_{6} \mathrm{D}_{5} \mathrm{CD}_{3}\right)$ of in situ generated $\mathrm{Cp}(\mathrm{IPr}) \mathrm{RuCl}\left(\eta^{2}-\mathrm{HSiCl}_{2} \mathrm{Me}\right)(\mathbf{4 b}) . \quad \mathrm{S} 7$

Figure S5. ${ }^{13} \mathrm{C}\left\{{ }^{1} \mathrm{H}\right\}$ NMR $\left(600 \mathrm{MHz}, 22^{\circ} \mathrm{C}\right.$, in $\left.\mathrm{C}_{6} \mathrm{D}_{6}\right)$ of $\mathrm{Cp}(\mathrm{IPr}) \mathrm{RuCl}\left(\eta^{2}-\mathrm{HSiCl}_{2} \mathrm{Me}\right)(\mathbf{4 b}) . \quad \mathrm{S} 8$

Figure S6. ${ }^{1} \mathrm{H}-{ }^{29} \mathrm{Si}$ INEPT NMR (119.2 MHz; $\left.-40^{\circ} \mathrm{C}, \mathrm{C}_{6} \mathrm{D}_{5} \mathrm{CD}_{3}\right)$ of $\mathrm{Cp}(\mathrm{IPr}) \mathrm{RuCl}\left(\eta^{2}-\mathrm{HSiCl}_{2} \mathrm{Me}\right)(\mathbf{4 b}) . \quad \mathrm{S} 8$

Figure S7. (a) ${ }^{1} \mathrm{H} \mathrm{NMR}\left(600 \mathrm{MHz}, 22{ }^{\circ} \mathrm{C}\right.$, in $\left.\mathrm{C}_{6} \mathrm{D}_{6}\right)$ of $\mathrm{Cp}(\mathrm{IPr}) \mathrm{RuCl}\left(\eta^{2}-\mathrm{HSiCl} \mathrm{Me}_{2}\right)(\mathbf{4 c})$; (b) ${ }^{1} \mathrm{H} \mathrm{NMR}$ $\left(600 \mathrm{MHz},-40^{\circ} \mathrm{C}, \mathrm{C}_{6} \mathrm{D}_{5} \mathrm{CD}_{3}\right)$ of in situ generated $\mathrm{Cp}(\mathrm{IPr}) \mathrm{RuCl}\left(\eta^{2}-\mathrm{HSiClMe}_{2}\right)(\mathbf{4 c})$.

Figure S8. ${ }^{13} \mathrm{C}\left\{{ }^{1} \mathrm{H}\right\}$ NMR $\left(600 \mathrm{MHz}, 22^{\circ} \mathrm{C}\right.$, in $\left.\mathrm{C}_{6} \mathrm{D}_{6}\right)$ of $\mathrm{Cp}(\mathrm{IPr}) \mathrm{RuCl}\left(\eta^{2}-\mathrm{HSiClMe}_{2}\right)(\mathbf{4 c}) . \quad \mathrm{S} 10$

Figure S9. ${ }^{1} \mathrm{H}-{ }^{29} \mathrm{Si}$ INEPT NMR (119.2 MHz, $\left.-40{ }^{\circ} \mathrm{C}, \mathrm{C}_{6} \mathrm{D}_{5} \mathrm{CD}_{3}\right)$ of $\mathrm{Cp}(\mathrm{IPr}) \mathrm{RuCl}\left(\eta^{2}-\mathrm{HSiClMe}_{2}\right)(\mathbf{4 c}) . \quad \mathrm{S} 10$

Figure S10. (a) ${ }^{1} \mathrm{H} \mathrm{NMR}\left(600 \mathrm{MHz}, 22^{\circ} \mathrm{C}, \mathrm{C}_{6} \mathrm{D}_{6}\right)$ of $\mathrm{Cp}(\mathrm{IPr}) \mathrm{RuCl}\left(\eta^{2}-\mathrm{H}_{3} \mathrm{SiPh}\right)(4 \mathrm{c})$; (b) ${ }^{1} \mathrm{H} \mathrm{NMR}(600$

$\left.\mathrm{MHz},-40^{\circ} \mathrm{C}, \mathrm{C}_{6} \mathrm{D}_{5} \mathrm{CD}_{3}\right)$ of in situ generated $\mathrm{Cp}(\mathrm{IPr}) \mathrm{RuCl}\left(\eta^{2}-\mathrm{H}_{3} \mathrm{SiPh}\right)(\mathbf{4 c})$.

Figure S11. ${ }^{13} \mathrm{C}\left\{{ }^{1} \mathrm{H}\right\}$ NMR $\left(600 \mathrm{MHz}, 22{ }^{\circ} \mathrm{C}, \mathrm{C}_{6} \mathrm{D}_{6}\right)$ of $\mathrm{Cp}(\mathrm{IPr}) \mathrm{RuCl}\left(\mathrm{HSiH}_{2} \mathrm{Ph}\right)(\mathbf{4 d}) . \quad \mathrm{S} 12$

Figure S12. ${ }^{1} \mathrm{H}-{ }^{29} \mathrm{Si}$ INEPT NMR (119.2 MHz, $\left.-40{ }^{\circ} \mathrm{C}, \mathrm{C}_{6} \mathrm{D}_{5} \mathrm{CD}_{3}\right)$ of $\mathrm{Cp}(\mathrm{IPr}) \mathrm{RuCl}\left(\eta^{2}-\mathrm{HSiH}_{2} \mathrm{Ph}\right)(\mathbf{4 d}) . \quad \mathrm{S} 12$

Figure S13. ${ }^{1} \mathrm{H} \mathrm{NMR}\left(600 \mathrm{MHz},-40^{\circ} \mathrm{C}, \mathrm{CD}_{2} \mathrm{Cl}_{2}\right)$ of in situ generated $\left[\mathrm{Cp}(\mathrm{IPr}) \mathrm{Ru}\left(\mathrm{NCCH}_{3}\right)\left(\eta^{2}-\mathrm{HSiCl}_{3}\right)\right] \mathrm{BArF}$

Figure S14. ${ }^{1} \mathrm{H}-{ }^{29} \mathrm{Si} \mathrm{HSQC}$ NMR $\left(119.2 \mathrm{MHz},-40^{\circ} \mathrm{C}, \mathrm{CD}_{2} \mathrm{Cl}_{2}\right)$ of $\left[\mathrm{Cp}(\mathrm{IPr}) \mathrm{Ru}\left(\mathrm{NCCH}_{3}\right)\left(\eta^{2}-\mathrm{HSiCl}_{3}\right)\right] \mathrm{BArF}$

Figure S15. ${ }^{1} \mathrm{H}-{ }^{29} \mathrm{Si}$ INEPT NMR $\left(119.2 \mathrm{MHz},-40^{\circ} \mathrm{C}, \mathrm{CD}_{2} \mathrm{Cl}_{2}\right)$ of $\left[\mathrm{Cp}(\mathrm{IPr}) \mathrm{Ru}\left(\mathrm{NCCH}_{3}\right)\left(\eta^{2}-\mathrm{HSiCl}_{3}\right)\right] \mathrm{BArF}$ 
Figure S16. ${ }^{1} \mathrm{H}-{ }^{29} \mathrm{Si}$ IHSQC NMR $\left(119.2 \mathrm{MHz},-40^{\circ} \mathrm{C}, \mathrm{CD}_{2} \mathrm{Cl}_{2}\right)$ of $\left[\mathrm{Cp}(\mathrm{IPr}) \mathrm{Ru}\left(\mathrm{NCCH}_{3}\right)\left(\eta^{2}-\mathrm{HSiH}_{2} \mathrm{Ph}\right)\right] \mathrm{BArF}$ (5d)

Figure S17. ${ }^{1} \mathrm{H}-{ }^{29} \mathrm{Si}$ INEPT NMR $\left(119.2 \mathrm{MHz},-40^{\circ} \mathrm{C}, \mathrm{CD}_{2} \mathrm{Cl}_{2}\right)$ of $\left[\mathrm{Cp}(\mathrm{IPr}) \mathrm{Ru}\left(\mathrm{NCCH}_{3}\right)\left(\eta^{2}-\mathrm{H}_{3} \mathrm{SiPh}\right)\right] \mathrm{BArF}(\mathbf{5 d})$

Figure S18. ${ }^{1} \mathrm{H}$ NMR $\left(600 \mathrm{MHz},-40^{\circ} \mathrm{C}, \mathrm{CD}_{2} \mathrm{Cl}_{2}\right)$ of $\left[\mathrm{Cp}(\mathrm{IPr}) \mathrm{Ru}\left(\mathrm{NCCH}_{3}\right)\left(\eta^{2}-\mathrm{HSiHMePh}\right)\right] \mathrm{BArF}$ (5e, two isomers).

Figure S19. ${ }^{1} \mathrm{H}-{ }^{29} \mathrm{Si}$ INEPT NMR $\left(119.2 \mathrm{MHz},-40^{\circ} \mathrm{C}, \mathrm{CD}_{2} \mathrm{Cl}_{2}\right)$ of $\left[\mathrm{Cp}(\mathrm{IPr}) \mathrm{Ru}\left(\mathrm{NCCH}_{3}\right)\left(\eta^{2}-\mathrm{HSiHMePh}\right)\right] \mathrm{BArF}(\mathbf{5 e})$

Figure S20. ${ }^{1} \mathrm{H}$ NMR $\left(400 \mathrm{MHz}, 22^{\circ} \mathrm{C}, \mathrm{C}_{6} \mathrm{D}_{6}\right)$ of $\mathrm{Cp}(\mathrm{IPr}) \mathrm{RuCl}(\mathbf{8})$.

Figure $\mathrm{S} 21 .{ }^{13} \mathrm{C}\left\{{ }^{1} \mathrm{H}\right\}$ NMR spectrum $\left(100.6 \mathrm{MHz}, 22^{\circ} \mathrm{C}, \mathrm{C}_{6} \mathrm{D}_{6}\right)$ of $\mathrm{Cp}(\mathrm{IPr}) \mathrm{RuCl}(\mathbf{8})$.

Figure S22. ${ }^{1} \mathrm{H}$ NMR $\left(600 \mathrm{MHz}, 22^{\circ} \mathrm{C}, \mathrm{NCCD}_{3}\right)$ of $\left[\mathrm{Cp}(\mathrm{IPr}) \mathrm{Ru}\left(\mathrm{NCCH}_{3}\right)_{2}\right] \mathrm{BArF}(9)$

Figure S23. ${ }^{13} \mathrm{C}\left\{{ }^{1} \mathrm{H}\right\}$ NMR spectrum $\left(151 \mathrm{MHz}, 22^{\circ} \mathrm{C}, \mathrm{NCCD}_{3}\right)$ of $\left[\mathrm{Cp}(\mathrm{IPr}) \mathrm{Ru}\left(\mathrm{NCCH}_{3}\right)_{2}\right] \mathrm{BArF}(\mathbf{9})$

Figure S24. ${ }^{11} \mathrm{~B}\left\{{ }^{1} \mathrm{H}\right\}$ NMR spectrum $(151 \mathrm{MHz}, \mathrm{NCCD} 3)$ of $\left[\mathrm{Cp}(\operatorname{IPr}) \mathrm{Ru}\left(\mathrm{NCCH}_{3}\right)_{2}\right] \mathrm{BArF}(\mathbf{9})$

Figure $\mathrm{S} 25 .{ }^{1} \mathrm{H}$ NMR $\left(600 \mathrm{MHz}, 22^{\circ} \mathrm{C}, \mathrm{CD}_{2} \mathrm{Cl}_{2}\right)$ of $[\mathrm{IPrH}]\left[\mathrm{CpRuCl}(\mathrm{H})\left(\mathrm{SiCl}_{3}\right)_{2}\right](\mathbf{1 0})$

Figure S26. ${ }^{13} \mathrm{C}\left\{{ }^{1} \mathrm{H}\right\}$ NMR $\left(600 \mathrm{MHz}, 22^{\circ} \mathrm{C}, \mathrm{CD}_{2} \mathrm{Cl}_{2}\right)$ of $[\mathrm{IPrH}]\left[\mathrm{CpRuCl}(\mathrm{H})\left(\mathrm{SiCl}_{3}\right)_{2}\right](\mathbf{1 0})$

Figure S27. ${ }^{1} \mathrm{H}-{ }^{29} \mathrm{Si} \mathrm{HSQC} \mathrm{NMR}\left(119.2 \mathrm{MHz}, 22^{\circ} \mathrm{C}, \mathrm{CD}_{2} \mathrm{Cl}_{2}\right)$ of $[\mathrm{IPrH}]\left[\mathrm{CpRuCl}(\mathrm{H})\left(\mathrm{SiCl}_{3}\right)_{2}\right](\mathbf{1 0})$ 
Table S1. Crystal and structure refinement data for $\mathbf{4 b}, \mathbf{4 d}, \mathbf{8}$, and $\mathbf{1 0}$

\begin{tabular}{|c|c|c|c|c|}
\hline & $4 \mathrm{bb}$ & $4 d$ & 8 & 10 \\
\hline Empirical formula & $\mathrm{C}_{33} \mathrm{H}_{45} \mathrm{Cl}_{3} \mathrm{~N}_{2} \mathrm{RuSi}$ & $\mathrm{C}_{76} \mathrm{H}_{98} \mathrm{Cl}_{2} \mathrm{~N}_{4} \mathrm{Ru}_{2} \mathrm{Si}_{2}$ & $\mathrm{C}_{32} \mathrm{H}_{41} \mathrm{ClN}_{2} \mathrm{Ru}$ & $\mathrm{C}_{32} \mathrm{H}_{43} \mathrm{Cl}_{7} \mathrm{~N}_{2} \mathrm{RuSi}_{2}$ \\
\hline Formula weight & 705.22 & 1396.80 & 590.19 & 861.08 \\
\hline colour, habit & yellow, block & yellow-red, block & blue, block & colourless, block \\
\hline Crystal size, $\mathrm{mm}^{3}$ & $0.28 \times 0.26 \times 0.24$ & $0.17 \times 0.11 \times 0.08$ & $0.11 \times 0.09 \times 0.06$ & $0.23 \times 0.18 \times 0.18$ \\
\hline Crystal system & Orthorhombic & triclinic & monoclinic & triclinic \\
\hline Space group & Pna2(1) & $\mathrm{P}-1$ & $\mathrm{P} 2(1) / \mathrm{c}$ & P-1 \\
\hline \multicolumn{5}{|l|}{ Unit cell dimensions: } \\
\hline $\mathrm{a}, \AA$ & $17.4593(11)$ & $11.4152(6)$ & $12.6112(3)$ & $11.2074(4)$ \\
\hline $\mathrm{b}, \AA$ & $12.4412(8)$ & $15.9638(9)$ & $14.8442(4)$ & $11.2257(4)$ \\
\hline $\mathrm{c}, \AA$ & $15.8779(10)$ & $19.8855(10)$ & $16.5695(4)$ & $18.4143(6)$ \\
\hline$\alpha$, deg. & 90 & 95.4893(17) & 90 & $83.2160(15)$ \\
\hline$\beta$, deg. & 90 & $98.2770(16)$ & $104.4642(14)$ & $88.0443(15)$ \\
\hline$\gamma$ deg. & 90 & $91.0324(16)$ & 90 & 60.9801(13) \\
\hline Volume, $\AA^{3}$ & $3448.9(4)$ & $3567.7(3)$ & $3003.55(13)$ & $2010.96(12)$ \\
\hline $\bar{Z}$ & 4 & 2 & 4 & 2 \\
\hline Density (calculated), $\mathrm{g} / \mathrm{cm}^{3}$ & 1.358 & 1.300 & 1.305 & 1.422 \\
\hline $\begin{array}{l}\text { Absorption coefficient, } \\
\mathrm{mm}^{-1}\end{array}$ & 0.746 & 0.576 & 0.633 & 0.939 \\
\hline $\mathrm{F}(000)$ & 1464 & 1464 & 1232 & 880 \\
\hline Diffractometer & Bruker SMART & Bruker SMART & Bruker SMART & Bruker SMART \\
\hline Temperature, $\mathrm{K}$ & $200(2)$ & $200(2)$ & $200(2)$ & $200(2)$ \\
\hline Radiation, (lambda, Å) & $(0.71073) \mathrm{Mo} \mathrm{K}_{\alpha}$ & $(0.71073)$ Mo K$_{\alpha}$ & $(0.71073) \mathrm{Mo} \mathrm{K}_{\alpha}$ & $(0.71073) \mathrm{Mo} \mathrm{K}_{\alpha}$ \\
\hline Scan mode & $\omega$ & $\omega$ & $\omega$ & $\omega$ \\
\hline Scan step (in omega), deg & 0.3 & 0.3 & 0.3 & 0.3 \\
\hline Time per step, sec. & 15 & 15 & 15 & 15 \\
\hline $\begin{array}{l}\text { Theta range for data } \\
\text { collection, deg }\end{array}$ & 2.01 to 28.32 & 1.28 to 28.33 & 1.87 to 28.32 & 1.11 to 28.35 \\
\hline Index ranges & $\begin{array}{l}-21 \leq h \leq 23,-16 \leq k \leq \\
16,-20 \leq l \leq 21\end{array}$ & $\begin{array}{l}-15 \leq h \leq 15,-21 \leq k \leq \\
21,-25 \leq l \leq 26\end{array}$ & $\begin{array}{l}-16 \leq h \leq 15,-19 \leq k \\
\leq 19,-22 \leq l \leq 22\end{array}$ & $\begin{array}{l}-14 \leq h \leq 13 ;-14 \leq k \leq \\
14 ;-24 \leq l \leq 24\end{array}$ \\
\hline Reflections collected & 25082 & 46453 & 43544 & 19434 \\
\hline Independent reflections & $\begin{array}{l}8210[\mathrm{R}(\text { int })= \\
0.0288]\end{array}$ & $\begin{array}{l}17610[\mathrm{R}(\mathrm{int})= \\
0.0318]\end{array}$ & $\begin{array}{l}7448[\mathrm{R}(\text { int })= \\
0.0503]\end{array}$ & $\begin{array}{l}9949[\mathrm{R}(\text { int })= \\
0.0389]\end{array}$ \\
\hline Absorption correction & multi-scan & multi-scan & multi-scan & multi-scan \\
\hline
\end{tabular}




\begin{tabular}{|l|l|l|l|l|}
\hline Max. transmission & 0.7457 & 0.955 & 0.7457 & 0.7457 \\
\hline Min. transmission & 0.6356 & 0.908 & 0.6838 & 0.6282 \\
\hline Refinement method & $\begin{array}{l}\text { Full-matrix least- } \\
\text { squares on F } \\
\text { (SHELXTL-Plus) }\end{array}$ & $\begin{array}{l}\text { Full-matrix least- } \\
\text { squares on F } \\
\text { (SHELXTL-Plus) }\end{array}$ & $\begin{array}{l}\text { Full-matrix least- } \\
\text { squares on F } \\
\text { (SHELXTL-Plus) }\end{array}$ & $\begin{array}{l}\text { Full-matrix least- } \\
\text { squares on } F^{2} \\
\text { (SHELXTL-Plus) }\end{array}$ \\
\hline $\begin{array}{l}\text { Data / restraints } \\
\text { parameters }\end{array}$ & $8210 / 1 / 361$ & $17610 / 0 / 755$ & $7448 / 0 / 325$ & $9949 / 0 / 397$ \\
\hline \begin{tabular}{l} 
Goodness-of-fit on F \\
\multicolumn{1}{|c|}{$\mathrm{R}$}
\end{tabular} & 1.030 & 1.010 & 1.021 & 1.063 \\
\hline $\begin{array}{l}\text { Final indices } \\
{[\mathrm{I}>2 \text { sigma(I)] }}\end{array}$ & $\begin{array}{l}\mathrm{R}_{1}=0.0296, \mathrm{wR}_{2}= \\
0.0662\end{array}$ & $\begin{array}{l}\mathrm{R}_{1}=0.0461, \mathrm{wR}_{2}= \\
0.1057\end{array}$ & $\begin{array}{l}\mathrm{R}_{1}=0.0352, \mathrm{wR}_{2} \\
=0.0775\end{array}$ & $\begin{array}{l}\mathrm{R}_{1}=0.0466, \mathrm{wR}_{2} \\
=0.1221\end{array}$ \\
\hline $\begin{array}{l}\mathrm{R} \text { indices (all data) } \\
\mathrm{R}_{1}=0.0329, \mathrm{wR}_{2}=\end{array}$ & $\begin{array}{l}\mathrm{R}_{1}=0.0735, \mathrm{wR}_{2}= \\
0.0678\end{array}$ & $\begin{array}{l}\mathrm{R}_{1}=0.0565, \mathrm{wR}_{2} \\
=0.0871\end{array}$ & $\begin{array}{l}\mathrm{R}_{1}=0.0755, \mathrm{wR}_{2} \\
=0.1291\end{array}$ \\
\hline $\begin{array}{l}\text { Largest diff. peak and hole, } \\
\text { e.A }\end{array}$ & $0.366,-0.400$ & $1.272,-1.214$ & $0.622,-0.493$ & $1.069,-0.586$ \\
\hline
\end{tabular}


$\mathrm{Cp}(\mathrm{IPr}) \mathrm{RuCl}\left(\eta^{2}-\mathrm{HSiCl}_{3}\right)(\mathbf{4 a})$

a)
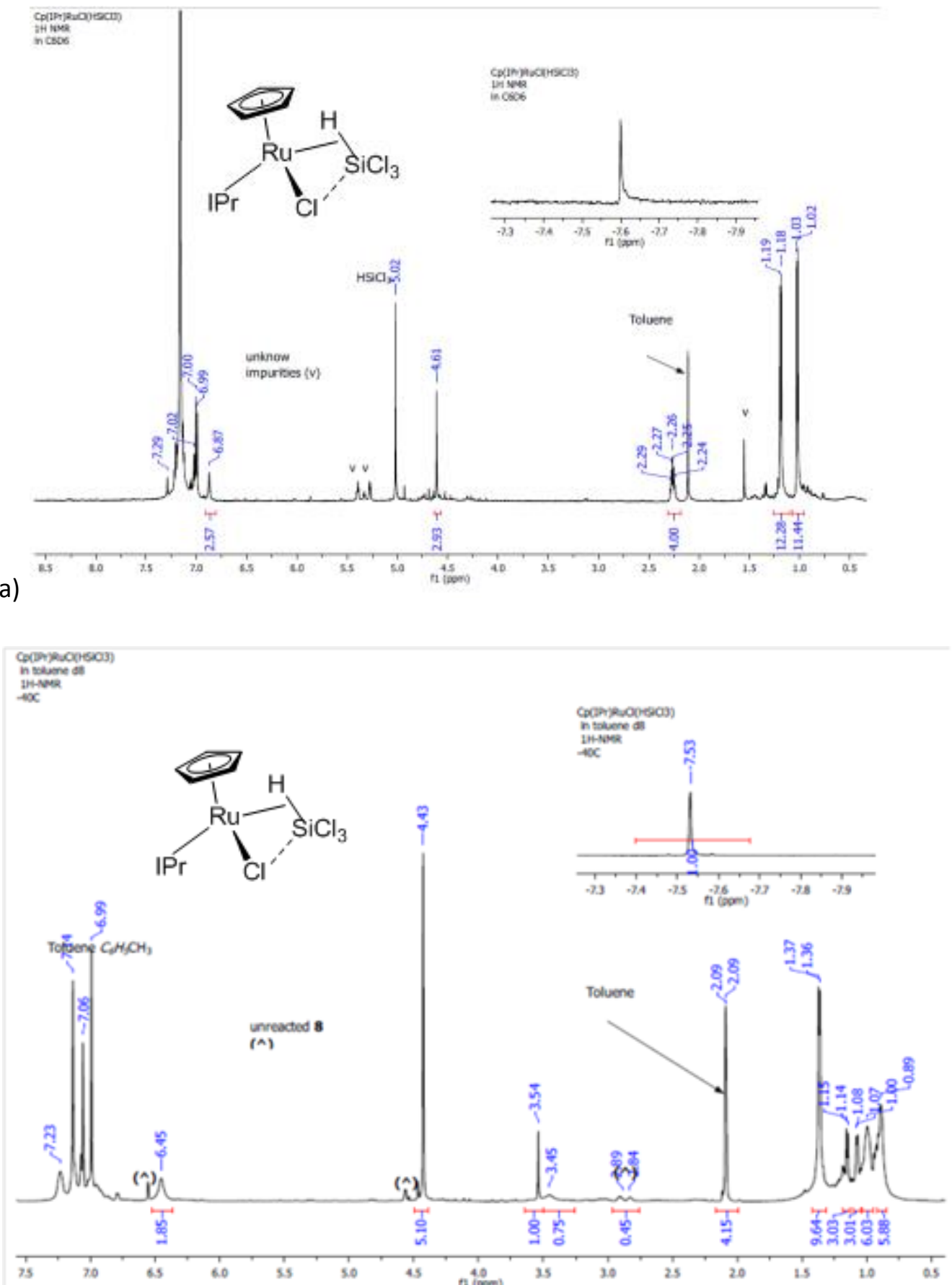

b)

Figure S1. (a) ${ }^{1} \mathrm{H}$ NMR $\left(600 \mathrm{MHz}, 22^{\circ} \mathrm{C}, \mathrm{C}_{6} \mathrm{D}_{6}\right)$ of $\mathrm{Cp}(\mathrm{IPr}) \mathrm{RuCl}\left(\eta^{2}-\mathrm{HSiCl}_{3}\right)(\mathbf{4 a})$; (b) ${ }^{1} \mathrm{H}$ NMR $(600 \mathrm{MHz},-40$ $\left.{ }^{\circ} \mathrm{C}, \mathrm{C}_{6} \mathrm{D}_{5} \mathrm{CD}_{3}\right)$ of in situ generated $\mathrm{Cp}(\operatorname{IPr}) \mathrm{RuCl}\left(\eta^{2}-\mathrm{HSiCl}_{3}\right)(\mathbf{4 a})$. 


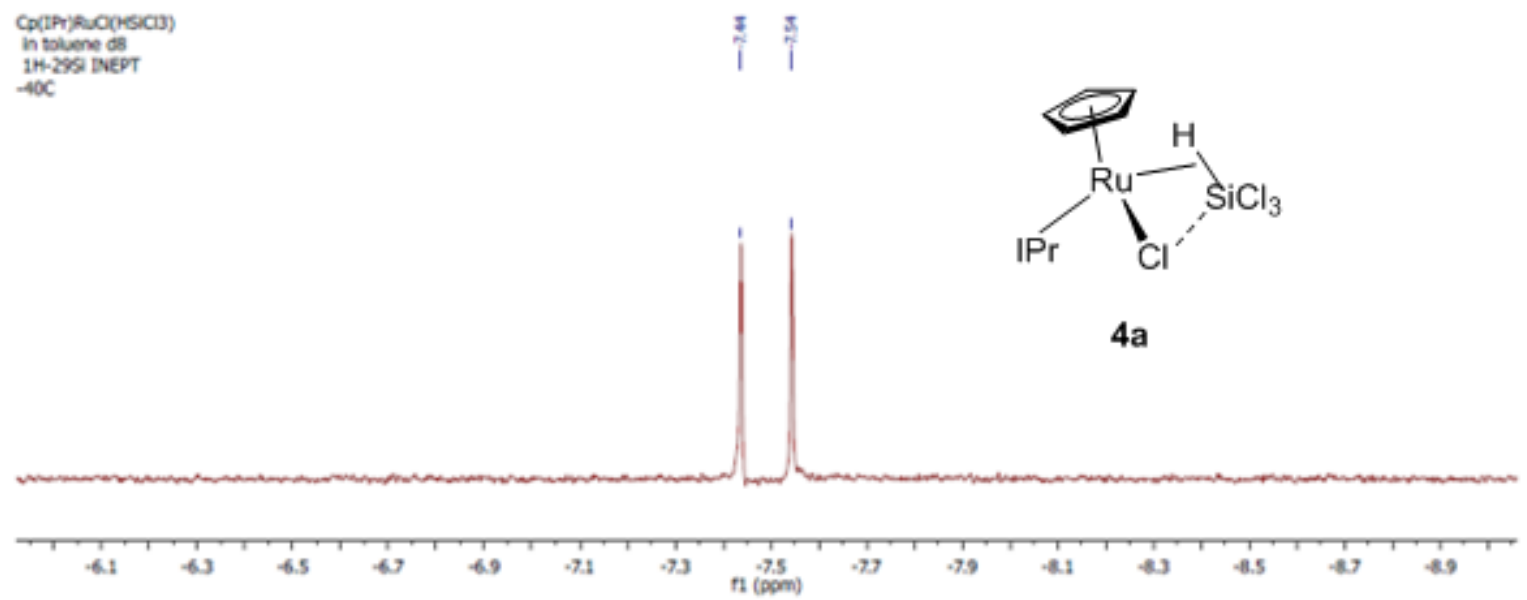

Figure S2. ${ }^{1} \mathrm{H}-{ }^{29} \mathrm{Si}$ INEPT NMR $\left(119.2 \mathrm{MHz},-40{ }^{\circ} \mathrm{C}, \mathrm{C}_{6} \mathrm{D}_{5} \mathrm{CD}_{3}\right)$ of $\mathrm{Cp}(\mathrm{IPr}) \mathrm{RuCl}\left(\eta^{2}-\mathrm{HSiCl}_{3}\right)(\mathbf{4 a})$

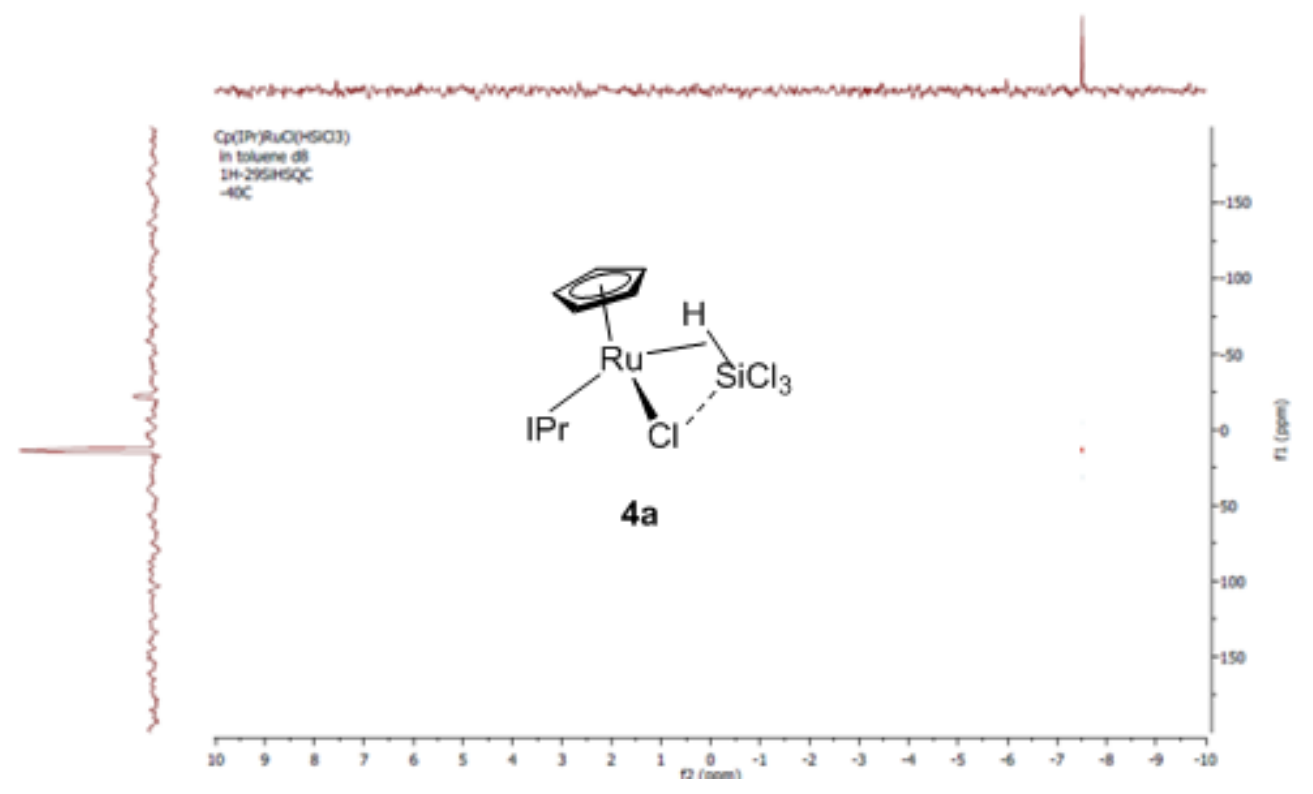

Figure S3. ${ }^{1} \mathrm{H}-{ }^{29} \mathrm{Si} \mathrm{HSQC} \mathrm{NMR}\left(119.2 \mathrm{MHz},-40{ }^{\circ} \mathrm{C}, \mathrm{C}_{6} \mathrm{D}_{5} \mathrm{CD}_{3}\right)$ of $\mathrm{Cp}(\mathrm{IPr}) \mathrm{RuCl}\left(\eta^{2}-\mathrm{HSiCl}_{3}\right)(\mathbf{4 a})$ 
$\mathrm{Cp}(\mathrm{IPr}) \mathrm{RuCl}\left(\eta^{2}-\mathrm{HSiCl}_{2} \mathrm{Me}\right)(\mathbf{4 b})$

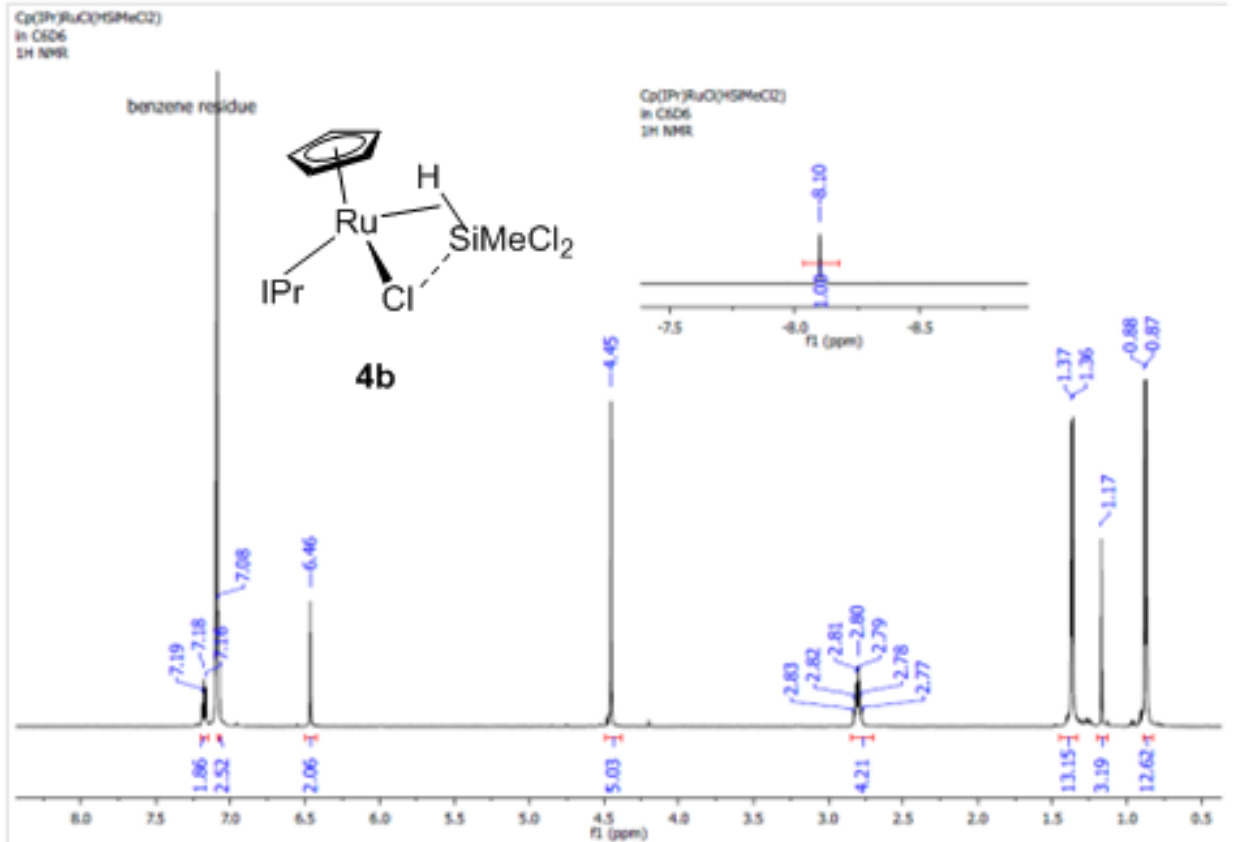

a)

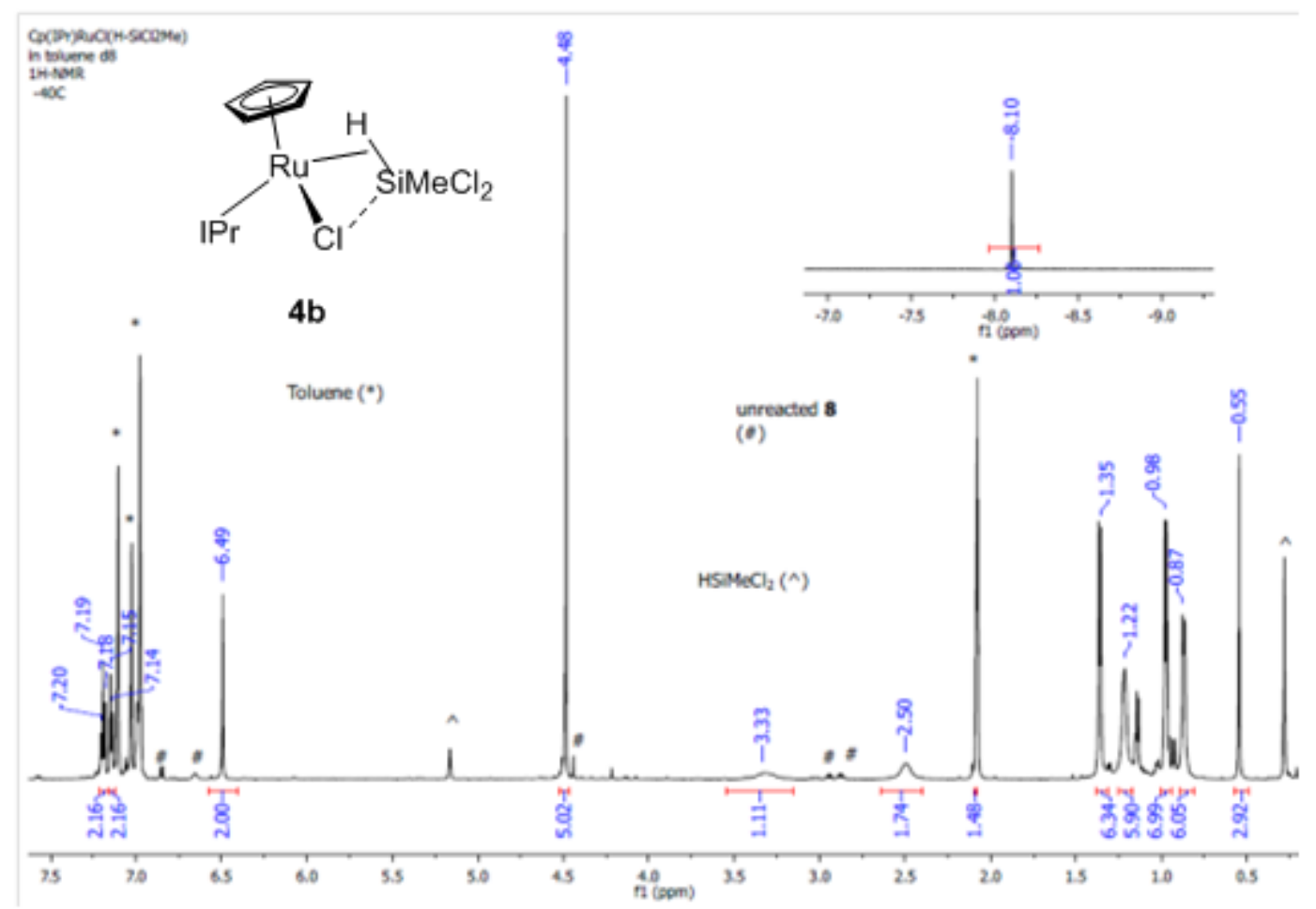

b)

Figure S4. (a) ${ }^{1} \mathrm{H}$ NMR $\left(600 \mathrm{MHz}, 22^{\circ} \mathrm{C}\right.$, in $\left.\mathrm{C}_{6} \mathrm{D}_{6}\right)$ of $\mathrm{Cp}(\mathrm{IPr}) \mathrm{RuCl}\left(\eta^{2}-\mathrm{HSiCl} \mathrm{Me}_{2} \mathrm{Me}(\mathbf{4 b})\right.$; (b) ${ }^{1} \mathrm{H} \mathrm{NMR}(600 \mathrm{MHz}$, $\left.-40^{\circ} \mathrm{C}, \mathrm{C}_{6} \mathrm{D}_{5} \mathrm{CD}_{3}\right)$ of in situ generated $\mathrm{Cp}(\mathrm{IPr}) \mathrm{RuCl}\left(\eta^{2}-\mathrm{HSiCl}_{2} \mathrm{Me}\right)(\mathbf{4 b})$. 


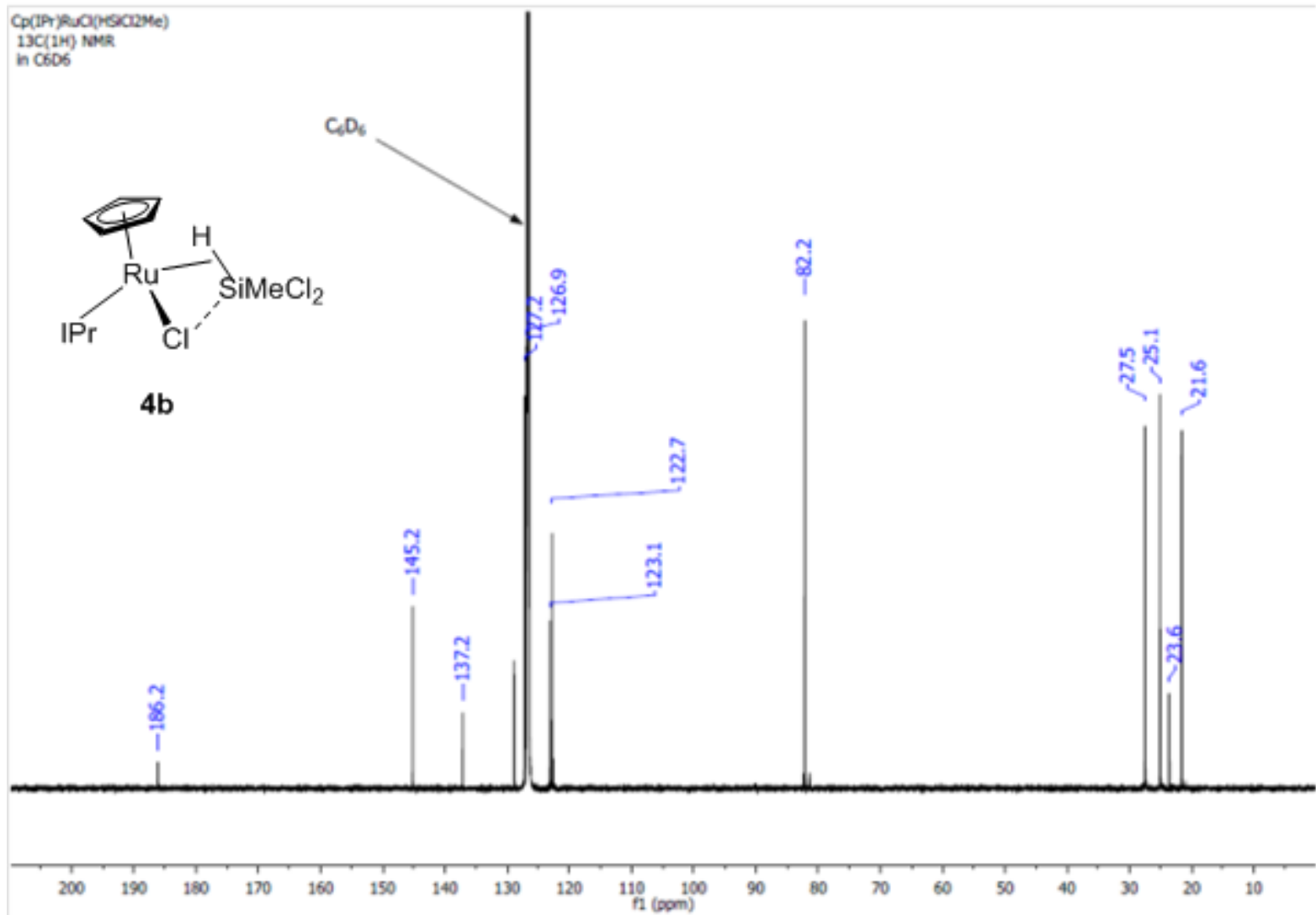

Figure S5. ${ }^{13} \mathrm{C}\left\{{ }^{1} \mathrm{H}\right\} \mathrm{NMR}\left(600 \mathrm{MHz}, 22^{\circ} \mathrm{C}\right.$, in $\left.\mathrm{C}_{6} \mathrm{D}_{6}\right)$ of $\mathrm{Cp}(\mathrm{IPr}) \mathrm{RuCl}\left(\eta^{2}-\mathrm{HSiCl}_{2} \mathrm{Me}\right)(\mathbf{4 b})$.

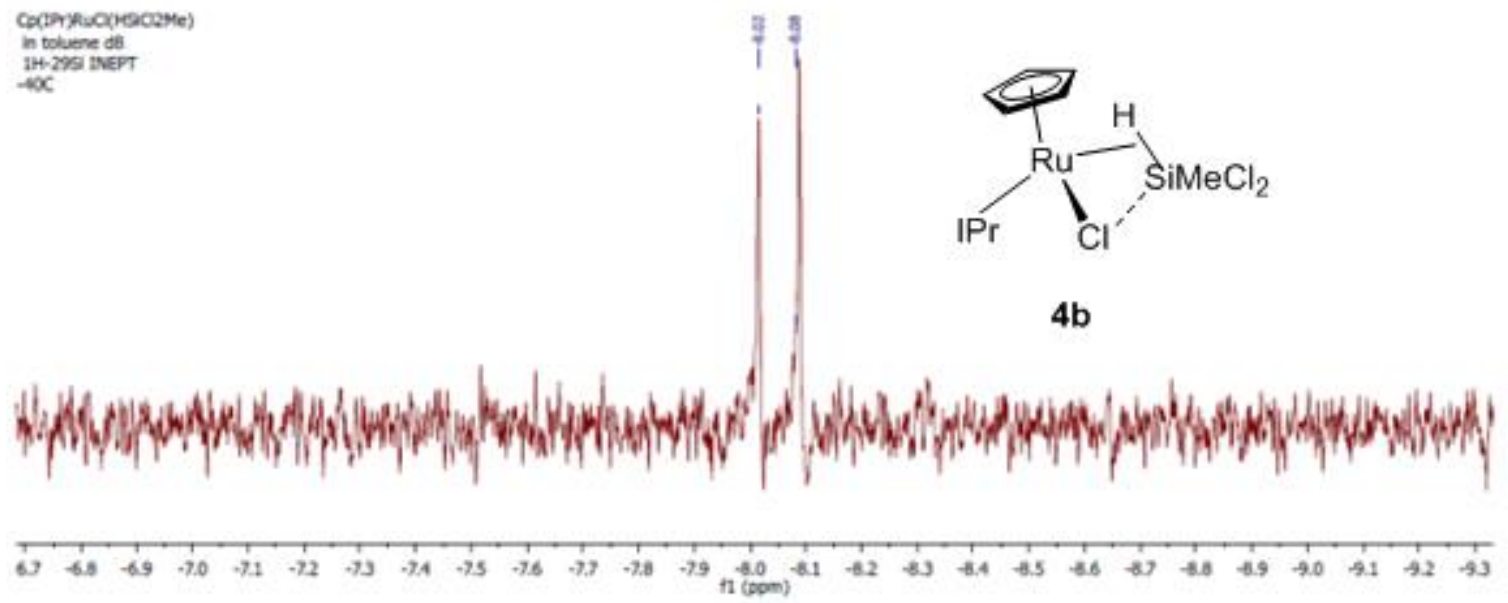

Figure S6. ${ }^{1} \mathrm{H}-{ }^{29} \mathrm{Si}$ INEPT NMR (119.2 MHz; $\left.-40{ }^{\circ} \mathrm{C}, \mathrm{C}_{6} \mathrm{D}_{5} \mathrm{CD}_{3}\right)$ of $\mathrm{Cp}(\mathrm{IPr}) \mathrm{RuCl}\left(\eta^{2}-\mathrm{HSiCl}_{2} \mathrm{Me}\right)(\mathbf{4 b})$. 
$\mathrm{Cp}(\mathrm{IPr}) \mathrm{RuCl}\left(\eta^{2}-\mathrm{HSiClMe}_{2}\right)(\mathbf{4 c})$

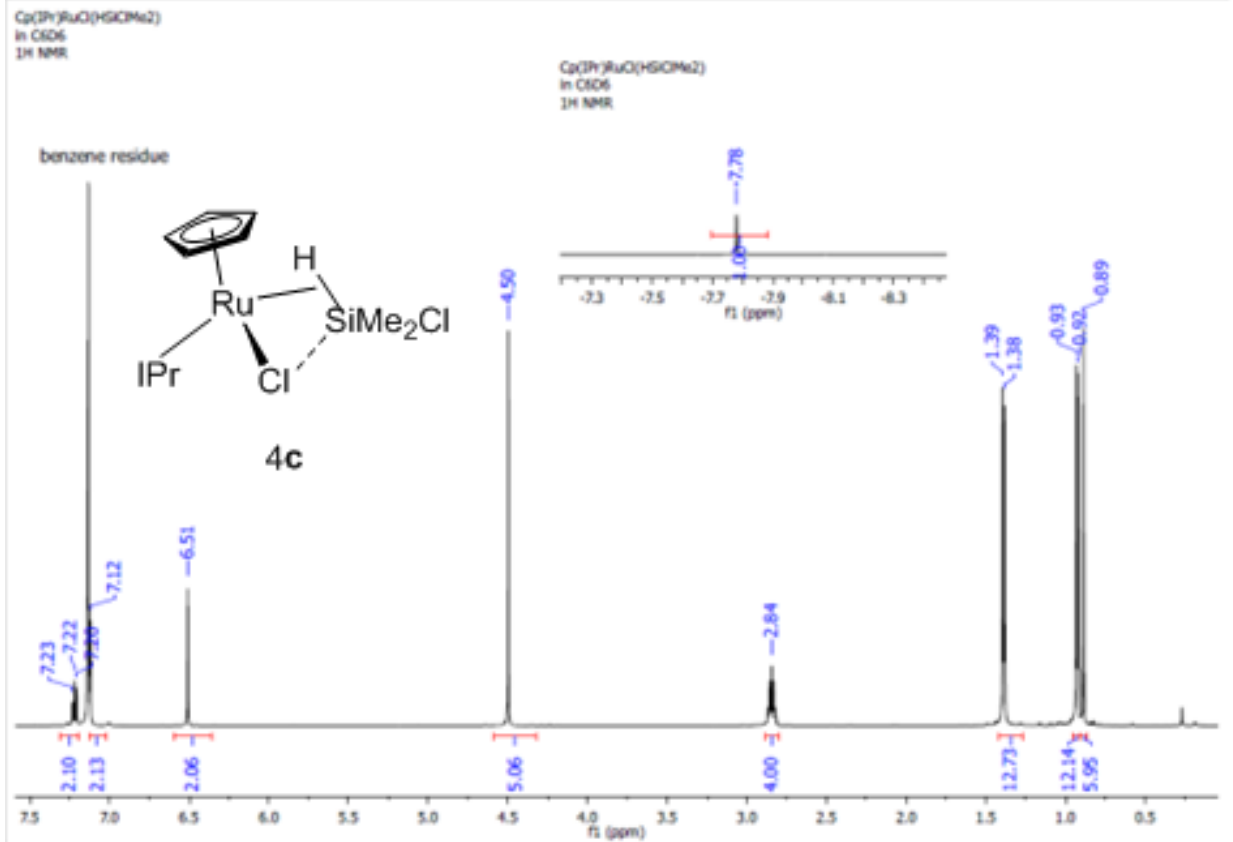

a)

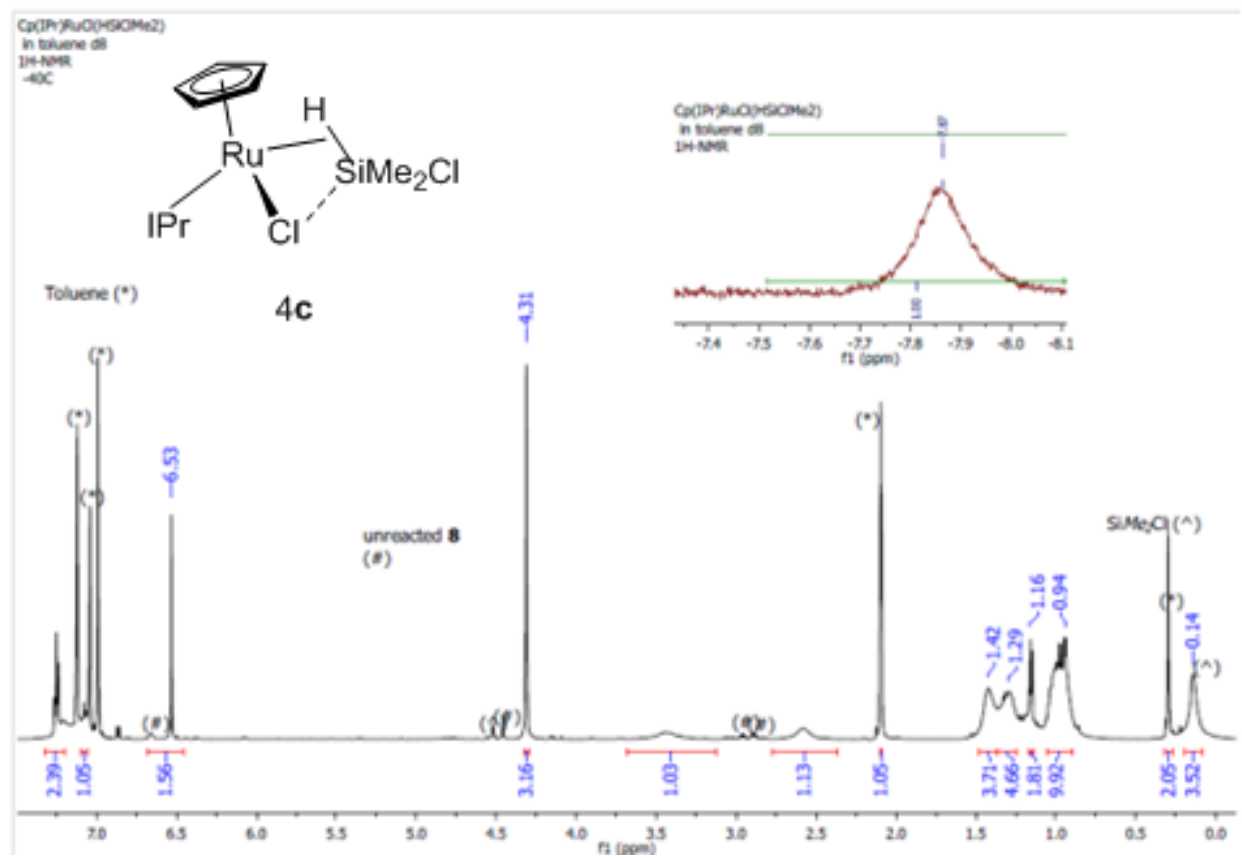

b)

Figure S7. (a) ${ }^{1} \mathrm{H}$ NMR $\left(600 \mathrm{MHz}, 22^{\circ} \mathrm{C}\right.$, in $\left.\mathrm{C}_{6} \mathrm{D}_{6}\right)$ of $\mathrm{Cp}(\mathrm{IPr}) \mathrm{RuCl}\left(\eta^{2}-\mathrm{HSiCl} \mathrm{Me}_{2}\right)(4 \mathrm{c})$; (b) ${ }^{1} \mathrm{H} \mathrm{NMR}(600$ $\left.\mathrm{MHz},-40^{\circ} \mathrm{C}, \mathrm{C}_{6} \mathrm{D}_{5} \mathrm{CD}_{3}\right)$ of in situ generated $\mathrm{Cp}(\mathrm{IPr}) \mathrm{RuCl}\left(\eta^{2}-\mathrm{HSiClMe}_{2}\right)(\mathbf{4 c})$. 


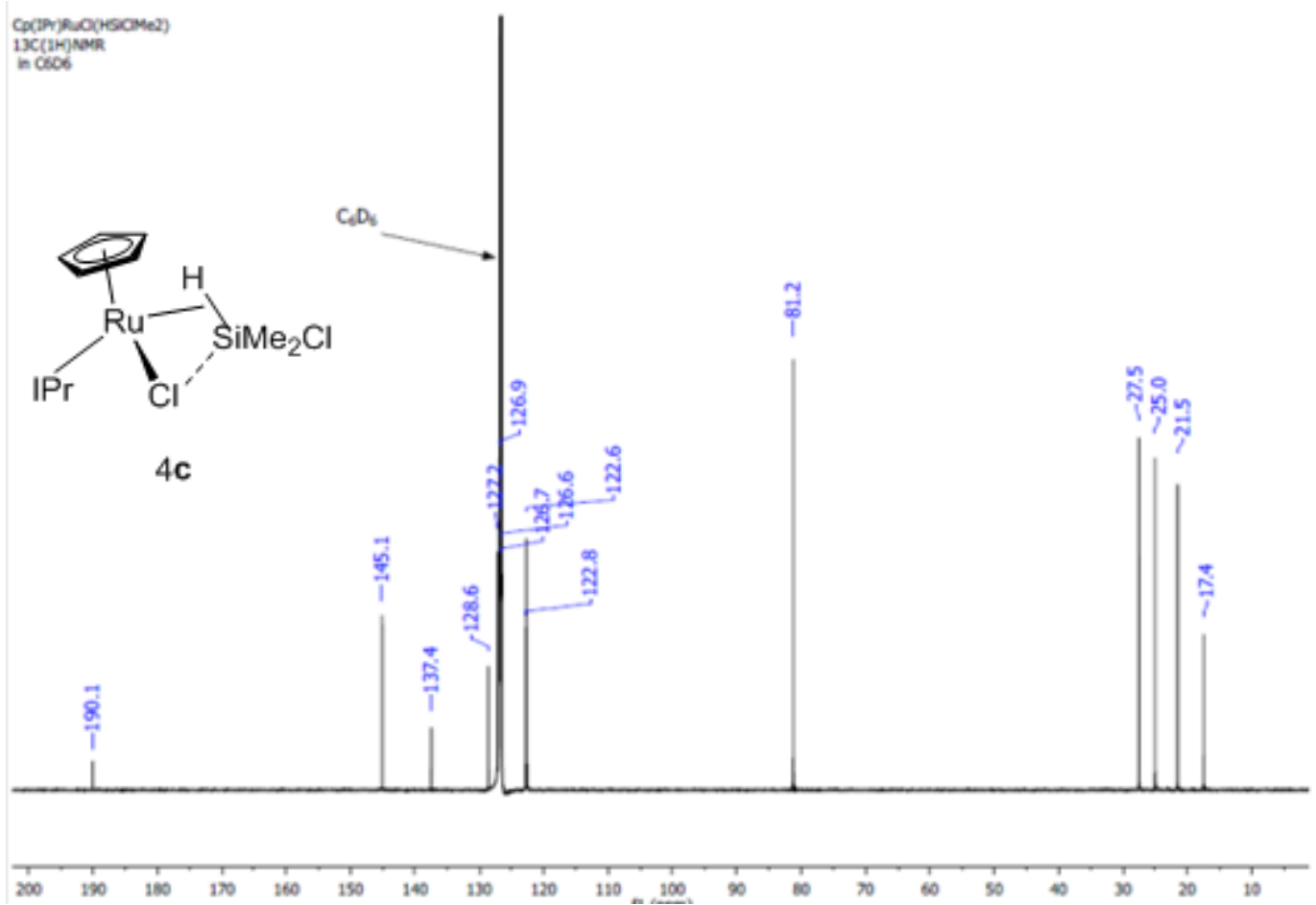

Figure S8. ${ }^{13} \mathrm{C}\left\{{ }^{1} \mathrm{H}\right\} \mathrm{NMR}\left(600 \mathrm{MHz}, 22^{\circ} \mathrm{C}\right.$, in $\left.\mathrm{C}_{6} \mathrm{D}_{6}\right)$ of $\mathrm{Cp}(\mathrm{IPr}) \mathrm{RuCl}\left(\eta^{2}-\mathrm{HSiClMe}_{2}\right)(4 \mathrm{c})$.

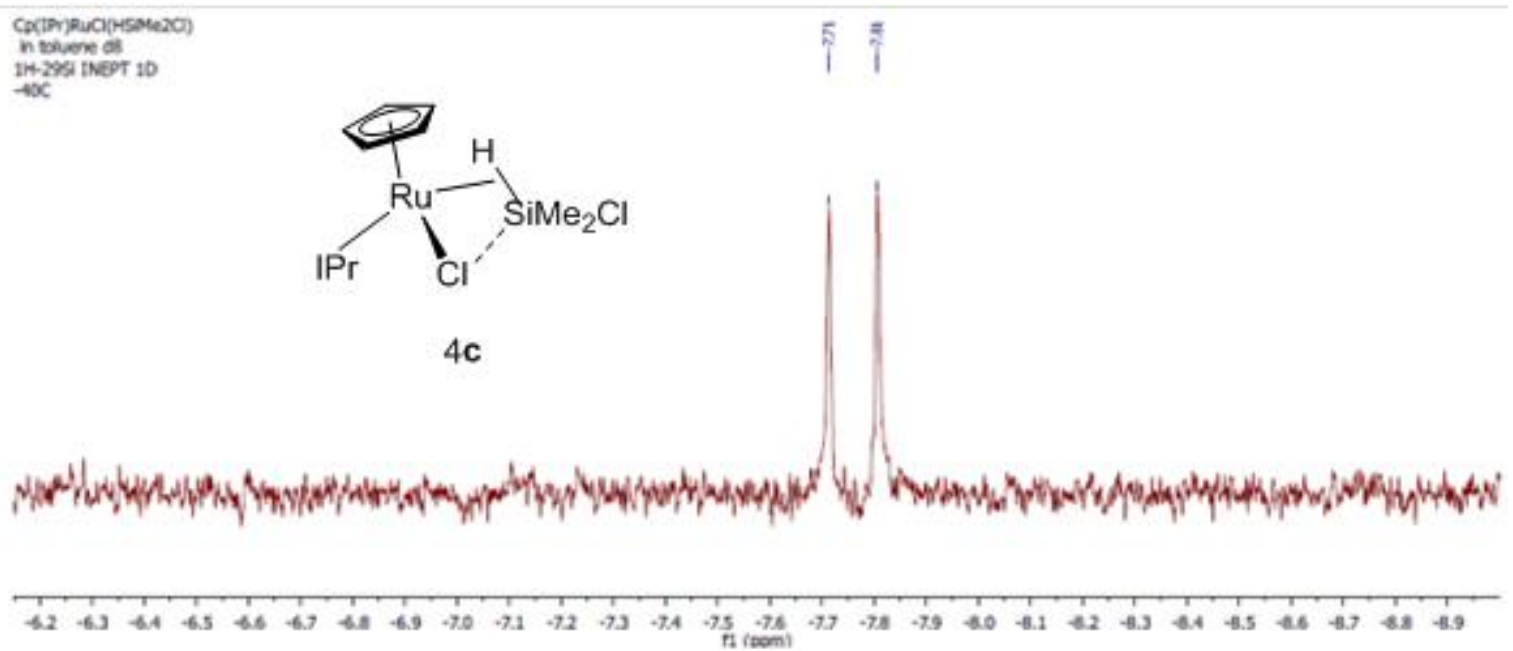

Figure S9. ${ }^{1} \mathrm{H}-{ }^{29} \mathrm{Si}$ INEPT NMR $\left(119.2 \mathrm{MHz},-40{ }^{\circ} \mathrm{C}, \mathrm{C}_{6} \mathrm{D}_{5} \mathrm{CD}_{3}\right)$ of $\mathrm{Cp}(\mathrm{IPr}) \mathrm{RuCl}\left(\eta^{2}-\mathrm{HSiClMe}_{2}\right)(4 \mathrm{c})$. 


\section{$\mathrm{Cp}(\mathrm{IPr}) \mathrm{RuCl}\left(\eta^{2}-\mathrm{HSiH}_{2} \mathrm{Ph}\right)(\mathbf{4 d})$}

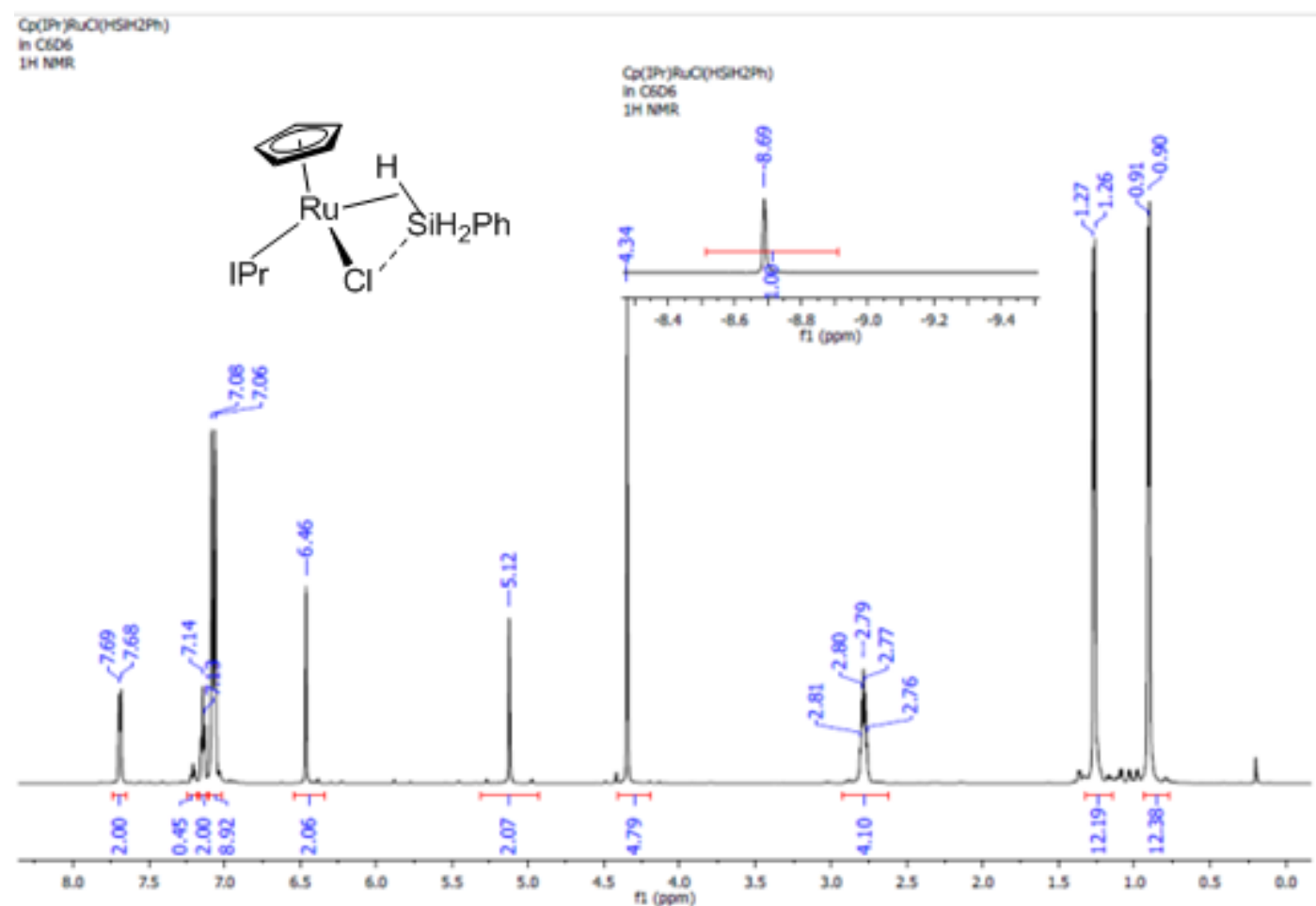

a)

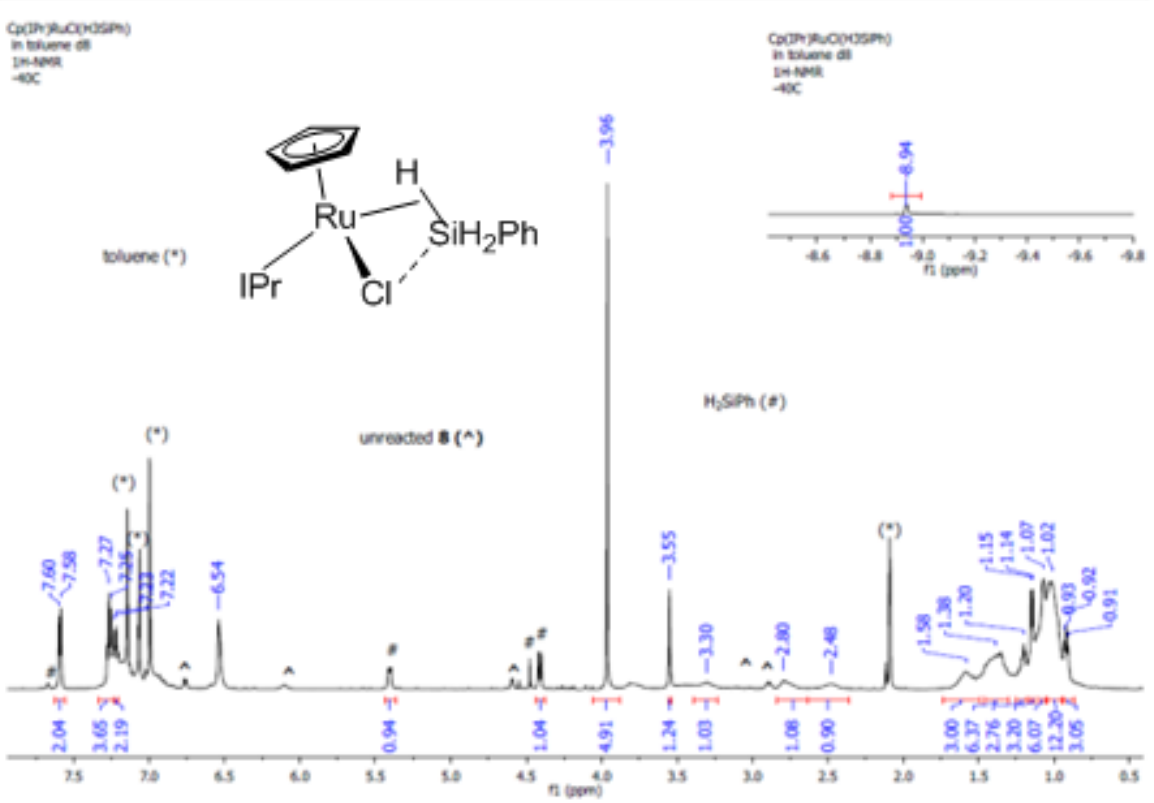

b)

Figure S10. (a) ${ }^{1} \mathrm{H}$ NMR $\left(600 \mathrm{MHz}, 22^{\circ} \mathrm{C}, \mathrm{C}_{6} \mathrm{D}_{6}\right)$ of $\mathrm{Cp}(\mathrm{IPr}) \mathrm{RuCl}\left(\eta^{2}-\mathrm{H}_{3} \mathrm{SiPh}\right)(4 \mathrm{c})$; (b) ${ }^{1} \mathrm{H} \mathrm{NMR}(600 \mathrm{MHz}$, $\left.40^{\circ} \mathrm{C}, \mathrm{C}_{6} \mathrm{D}_{5} \mathrm{CD}_{3}\right)$ of in situ generated $\mathrm{Cp}(\operatorname{IPr}) \mathrm{RuCl}\left(\eta^{2}-\mathrm{H}_{3} \mathrm{SiPh}\right)(\mathbf{4 c})$. 


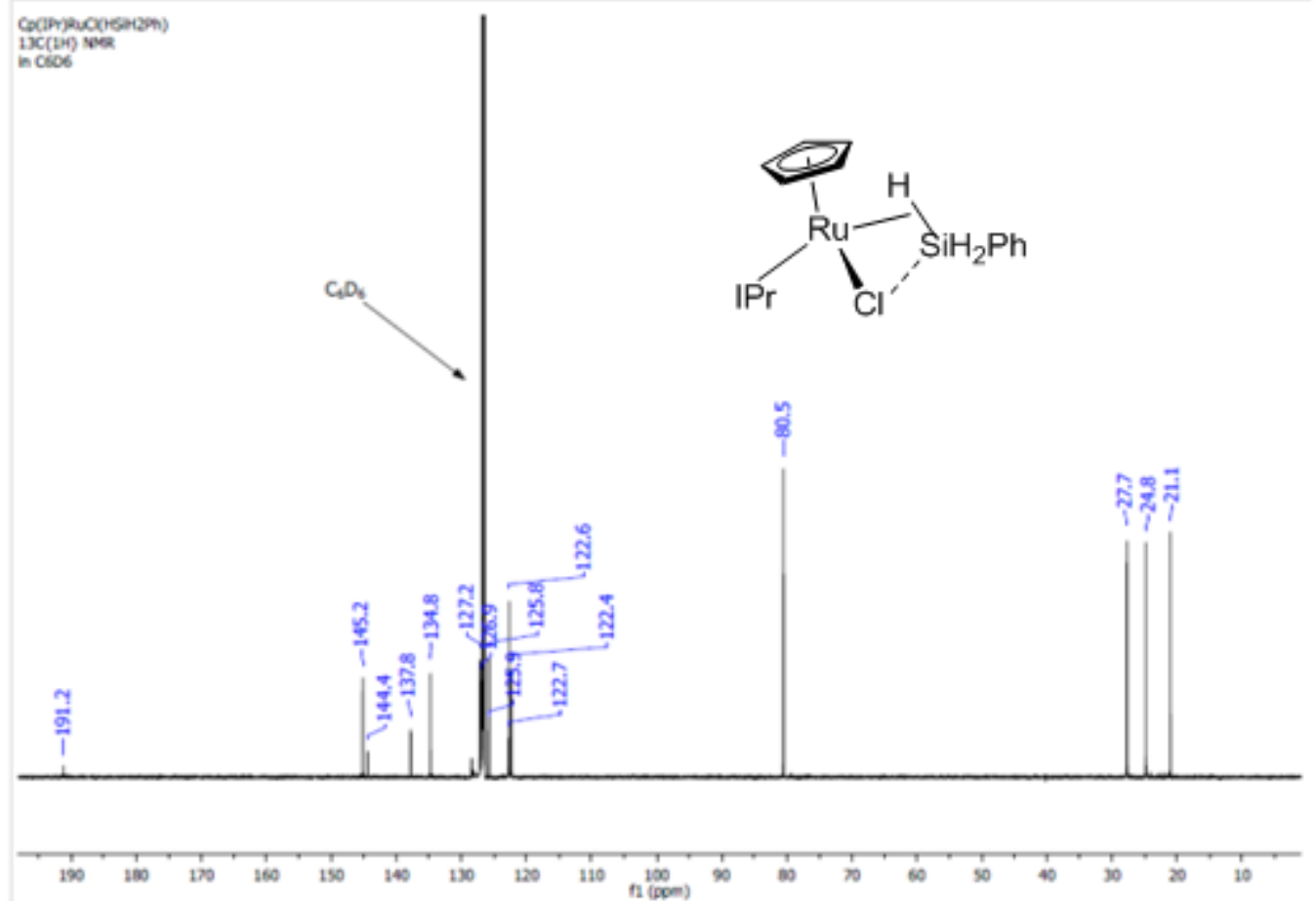

Figure S11. ${ }^{13} \mathrm{C}\left\{{ }^{1} \mathrm{H}\right\}$ NMR $\left(600 \mathrm{MHz}, 22^{\circ} \mathrm{C}, \mathrm{C}_{6} \mathrm{D}_{6}\right)$ of $\mathrm{Cp}(\mathrm{IPr}) \mathrm{RuCl}\left(\mathrm{HSiH}_{2} \mathrm{Ph}\right)(\mathbf{4 d})$.

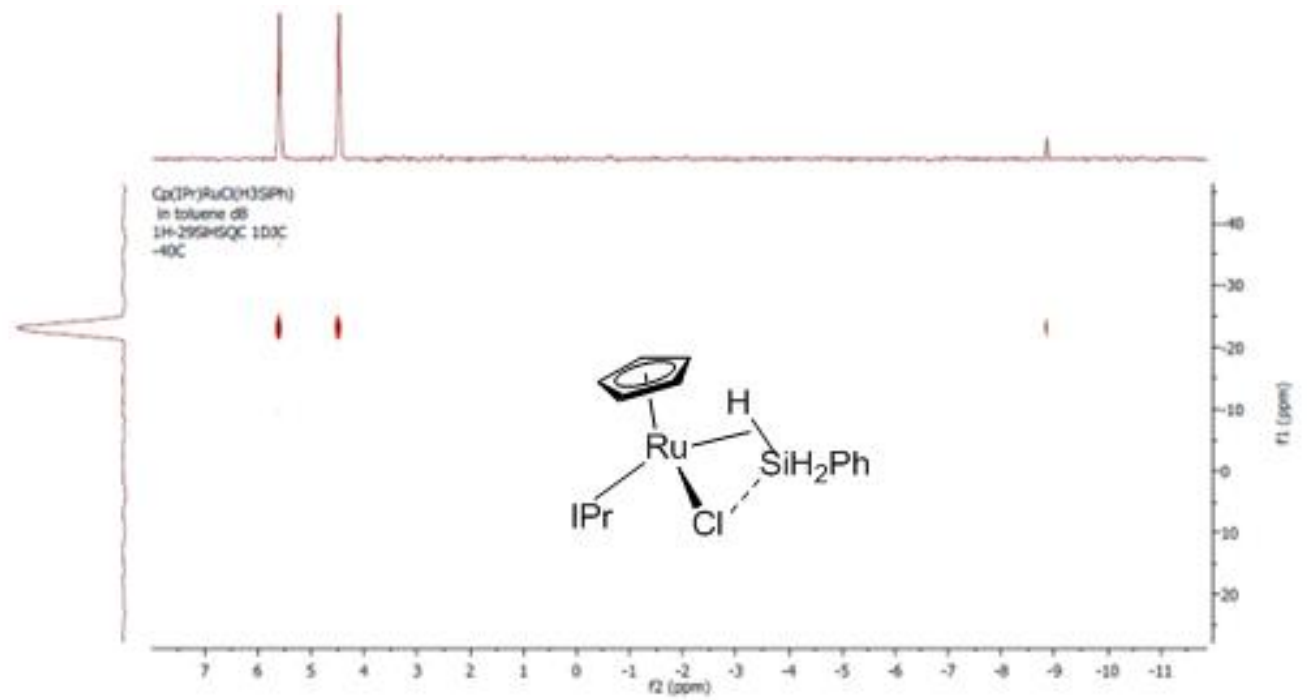

Figure S12. ${ }^{1} \mathrm{H}-{ }^{29} \mathrm{Si}$ INEPT NMR $\left(119.2 \mathrm{MHz},-40{ }^{\circ} \mathrm{C}, \mathrm{C}_{6} \mathrm{D}_{5} \mathrm{CD}_{3}\right)$ of $\mathrm{Cp}(\mathrm{IPr}) \mathrm{RuCl}\left(\eta^{2}-\mathrm{HSiH}_{2} \mathrm{Ph}\right)(\mathbf{4 d})$ 
$\left[\mathrm{Cp}(\mathrm{IPr}) \mathrm{Ru}\left(\mathrm{NCCH}_{3}\right)\left(\eta^{2}-\mathrm{HSiCl}_{3}\right)\right] \mathrm{BArF}(\mathbf{5 a})$

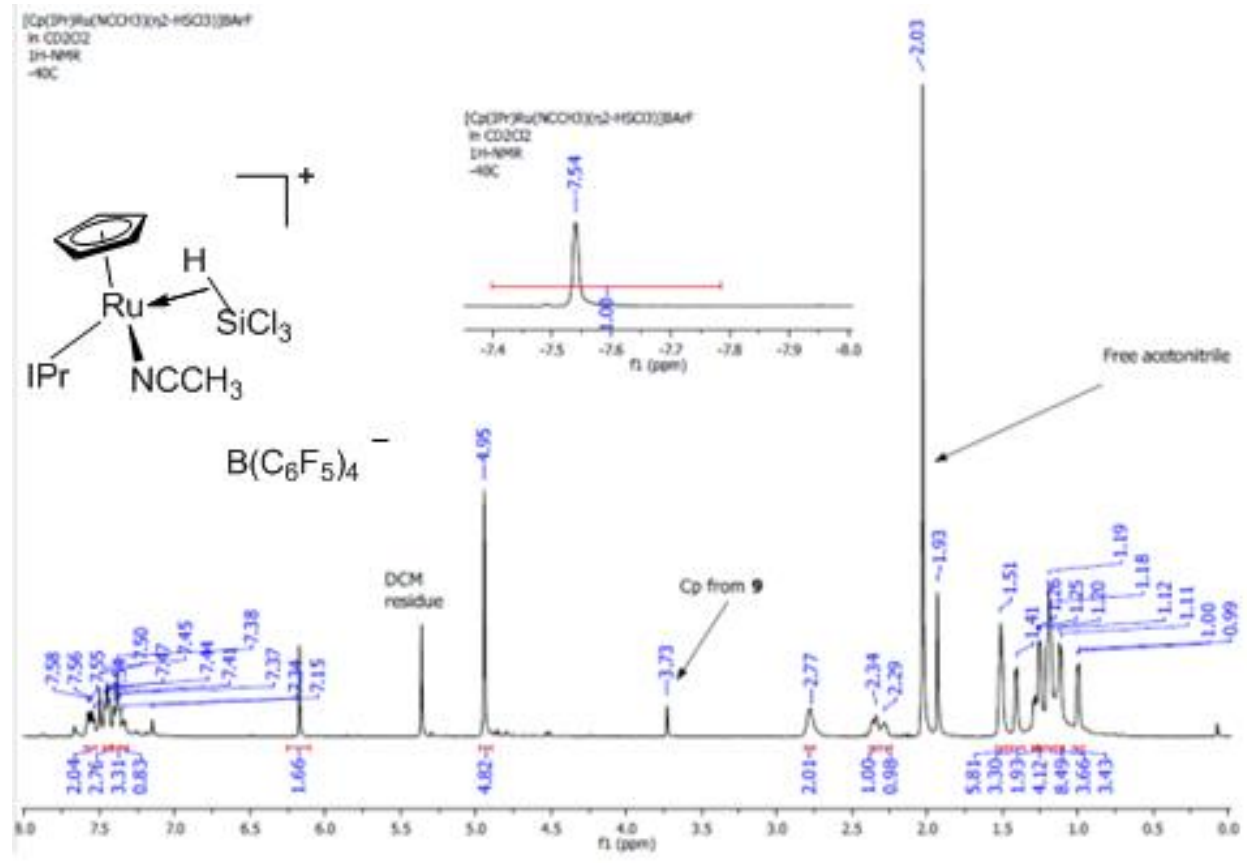

Figure S13. ${ }^{1} \mathrm{H}$ NMR $\left(600 \mathrm{MHz},-40^{\circ} \mathrm{C}, \mathrm{CD}_{2} \mathrm{Cl}_{2}\right)$ of in situ generated $\left[\mathrm{Cp}(\mathrm{IPr}) \mathrm{Ru}\left(\mathrm{NCCH}_{3}\right)\left(\eta^{2}-\mathrm{HSiCl}_{3}\right)\right] \mathrm{BArF}$ (5a)

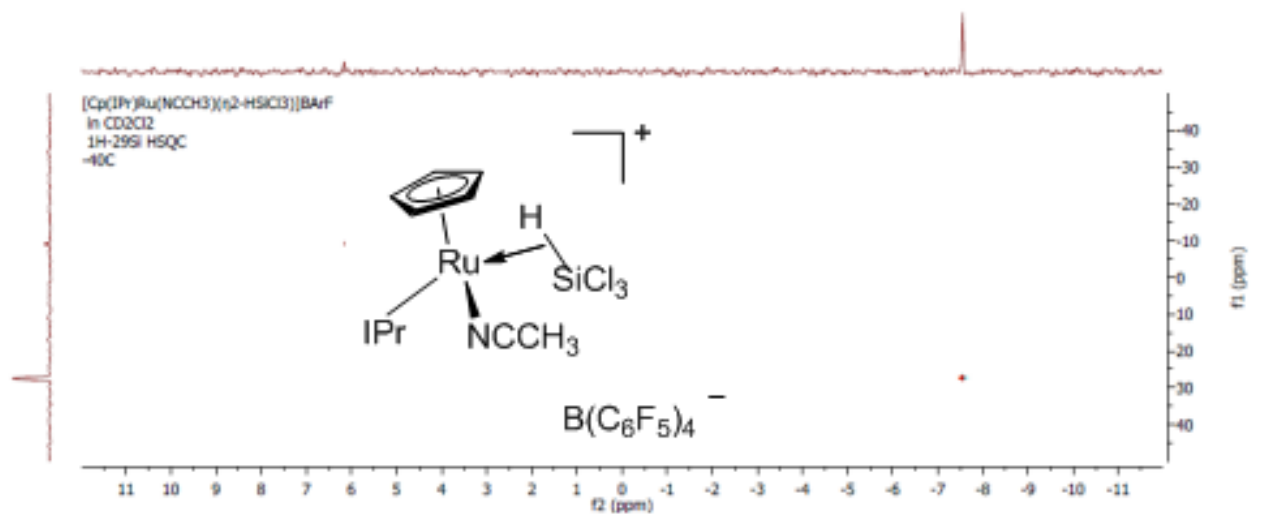

Figure S14. ${ }^{1} \mathrm{H}-{ }^{29} \mathrm{Si} \mathrm{HSQC} \mathrm{NMR}\left(119.2 \mathrm{MHz},-40^{\circ} \mathrm{C}, \mathrm{CD}_{2} \mathrm{Cl}_{2}\right)$ of $\left[\mathrm{Cp}(\mathrm{IPr}) \mathrm{Ru}\left(\mathrm{NCCH}_{3}\right)\left(\eta^{2}-\mathrm{HSiCl}_{3}\right)\right] \mathrm{BArF}(\mathbf{5 a})$
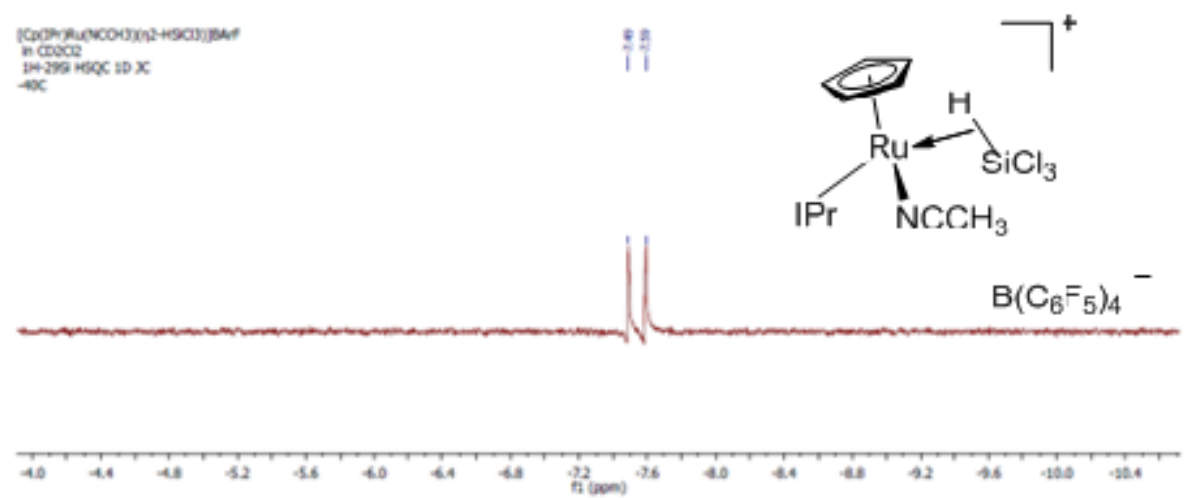

Figure $\mathrm{S} 15 .{ }^{1} \mathrm{H}-{ }^{29} \mathrm{Si}$ INEPT NMR $\left(119.2 \mathrm{MHz},-40^{\circ} \mathrm{C}, \mathrm{CD}_{2} \mathrm{Cl}_{2}\right)$ of $\left[\mathrm{Cp}(\mathrm{IPr}) \mathrm{Ru}\left(\mathrm{NCCH}_{3}\right)\left(\eta^{2}-\mathrm{HSiCl}_{3}\right)\right] \mathrm{BArF}(\mathbf{5 a})$ 
$\left[\mathrm{Cp}(\mathrm{IPr}) \mathrm{Ru}\left(\mathrm{NCCH}_{3}\right)\left(\eta^{2}-\mathrm{HSiH}_{2} \mathrm{Ph}\right)\right] \mathrm{BArF}(\mathbf{5 d})$

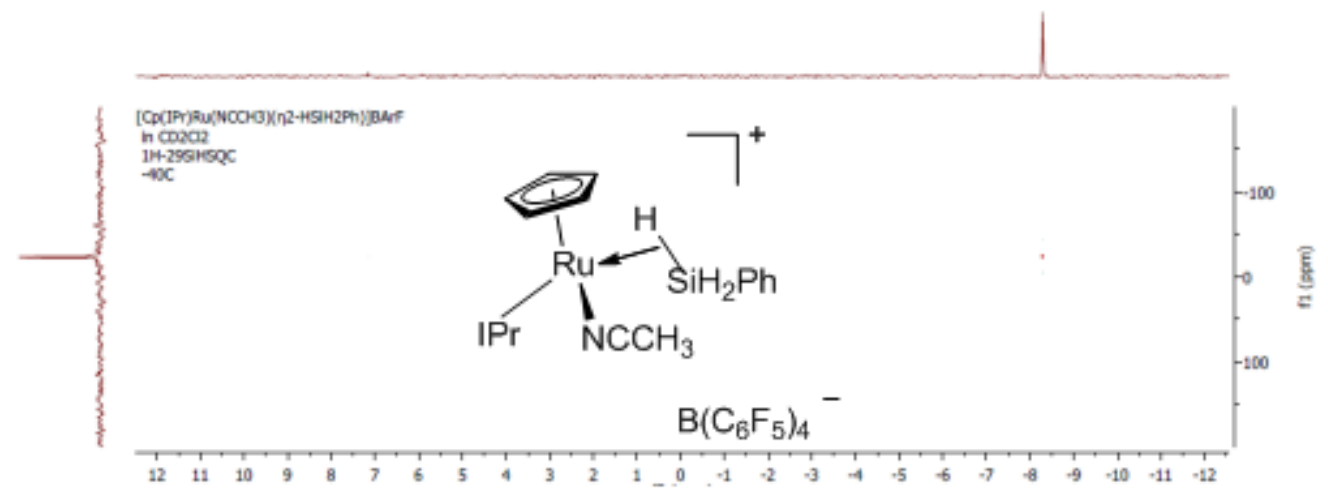

Figure S16. ${ }^{1} \mathrm{H}-{ }^{29} \mathrm{Si}$ IHSQC NMR $\left(119.2 \mathrm{MHz},-40^{\circ} \mathrm{C}, \mathrm{CD}_{2} \mathrm{Cl}_{2}\right)$ of $\left[\mathrm{Cp}(\mathrm{IPr}) \mathrm{Ru}\left(\mathrm{NCCH}_{3}\right)\left(\eta^{2}-\mathrm{H}_{2} \mathrm{SiPh}\right)\right] \mathrm{BArF}(\mathbf{5 d})$
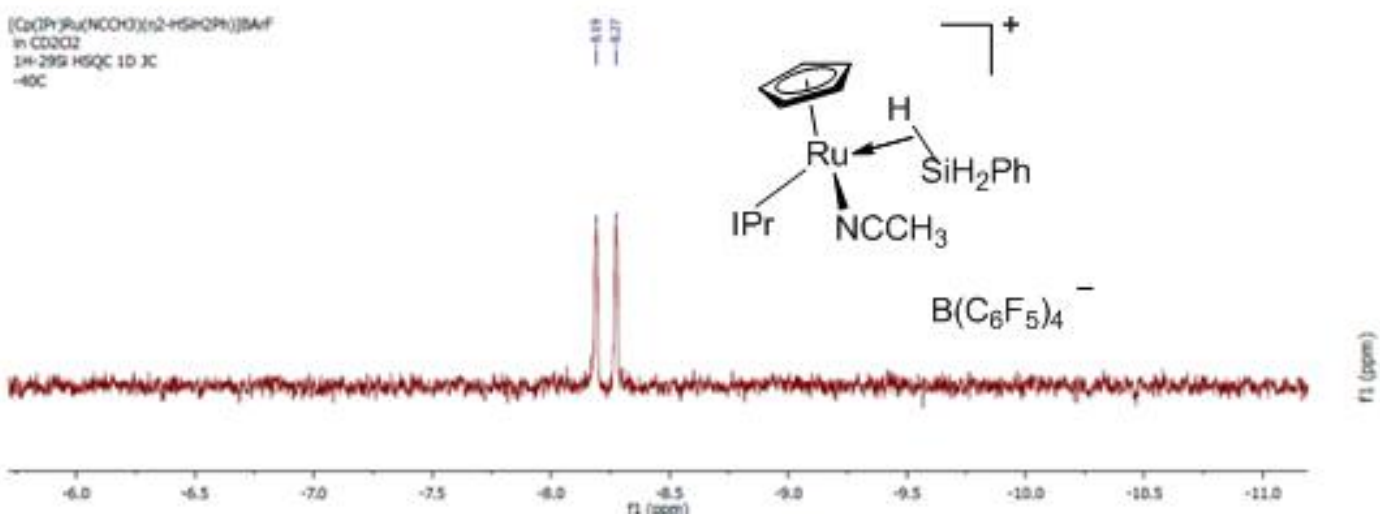

Figure S17. ${ }^{1} \mathrm{H}-{ }^{29} \mathrm{Si}$ INEPT NMR $\left(119.2 \mathrm{MHz},-40{ }^{\circ} \mathrm{C}, \mathrm{CD}_{2} \mathrm{Cl}_{2}\right)$ of $\left[\mathrm{Cp}(\mathrm{IPr}) \mathrm{Ru}\left(\mathrm{NCCH}_{3}\right)\left(\eta^{2}-\mathrm{H}_{3} \mathrm{SiPh}\right)\right] \mathrm{BArF}(\mathbf{5 d})$ 
$\left[\mathrm{Cp}(\mathrm{IPr}) \mathrm{Ru}\left(\mathrm{NCCH}_{3}\right)\left(\eta^{2}-\mathrm{HSiHMePh}\right)\right] \mathrm{BArF}(\mathbf{5 e})$
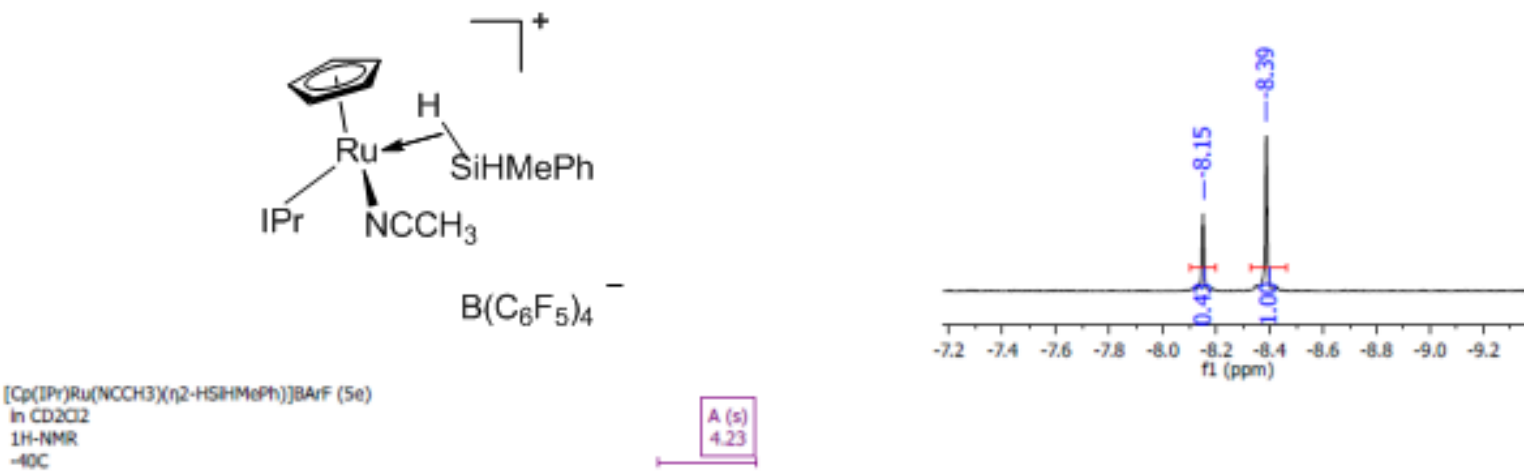

\section{\begin{tabular}{l}
$\mathrm{A}(\mathrm{s})$ \\
4.23 \\
\hline
\end{tabular}}

$$
\rightarrow+0 \mathrm{C}
$$

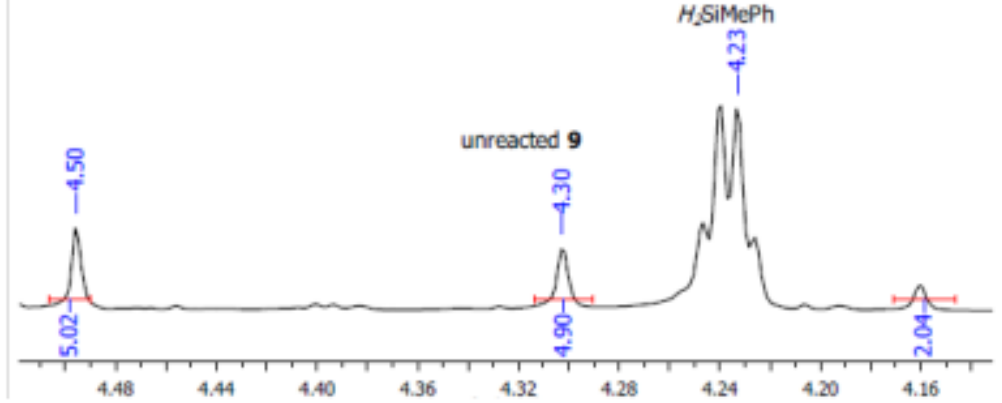

Figure S18. ${ }^{1} \mathrm{H}$ NMR $\left(600 \mathrm{MHz},-40^{\circ} \mathrm{C}, \mathrm{CD}_{2} \mathrm{Cl}_{2}\right)$ of in situ generated $\left[\mathrm{Cp}(\mathrm{IPr}) \mathrm{Ru}\left(\mathrm{NCCH}_{3}\right)\left(\eta^{2}-\right.\right.$ $\mathrm{HSiHMePh})] \mathrm{BArF}$ (5e, two isomers).

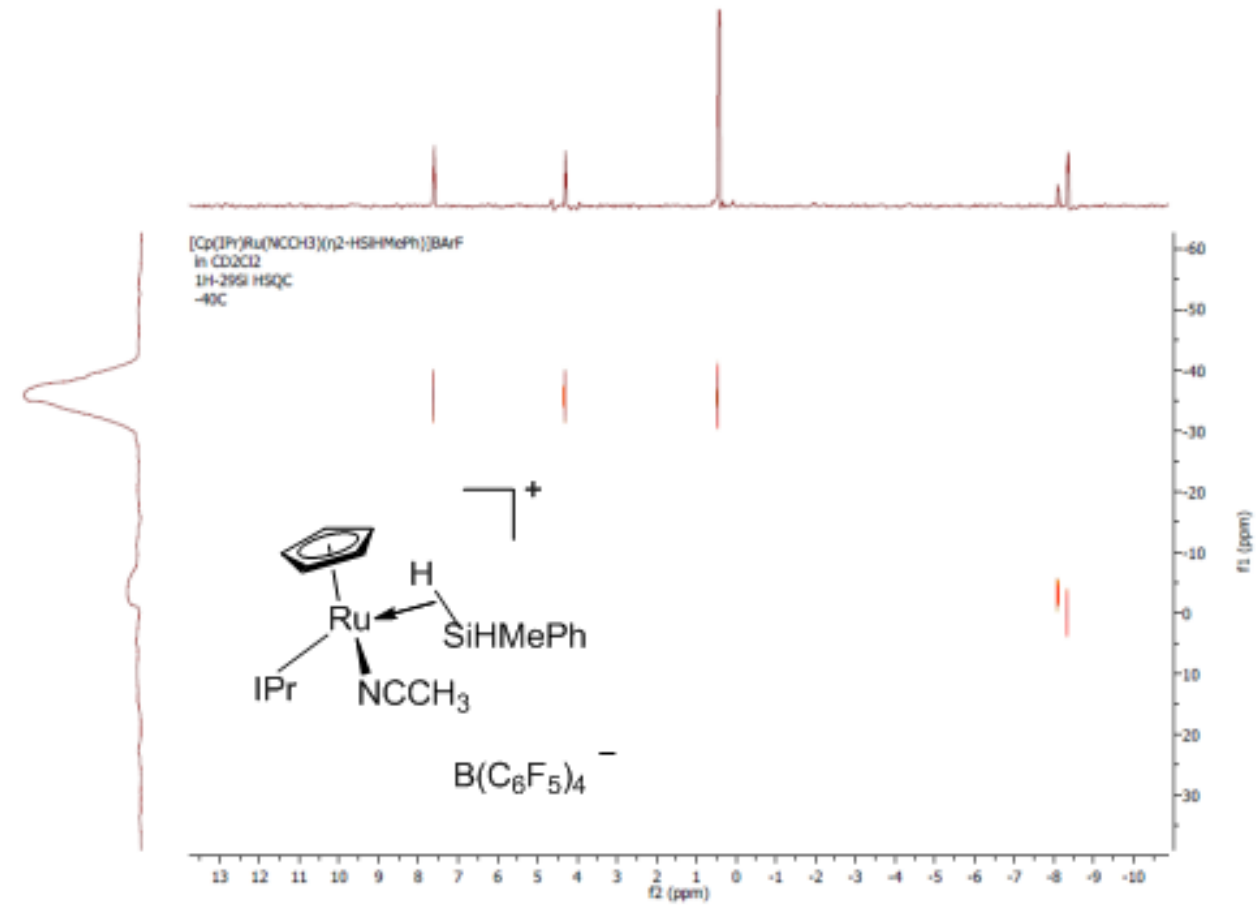

Figure S19. ${ }^{1} \mathrm{H}-{ }^{29} \mathrm{Si}$ INEPT NMR $\left(119.2 \mathrm{MHz},-40^{\circ} \mathrm{C}, \mathrm{CD}_{2} \mathrm{Cl}_{2}\right)$ of $\left[\mathrm{Cp}(\mathrm{IPr}) \mathrm{Ru}\left(\mathrm{NCCH}_{3}\right)\left(\eta^{2}-\mathrm{HSiHMePh}\right)\right] \mathrm{BArF}$ (5e) 
$\mathrm{Cp}(\mathrm{IPr}) \mathrm{RuCl}(\mathbf{8})$

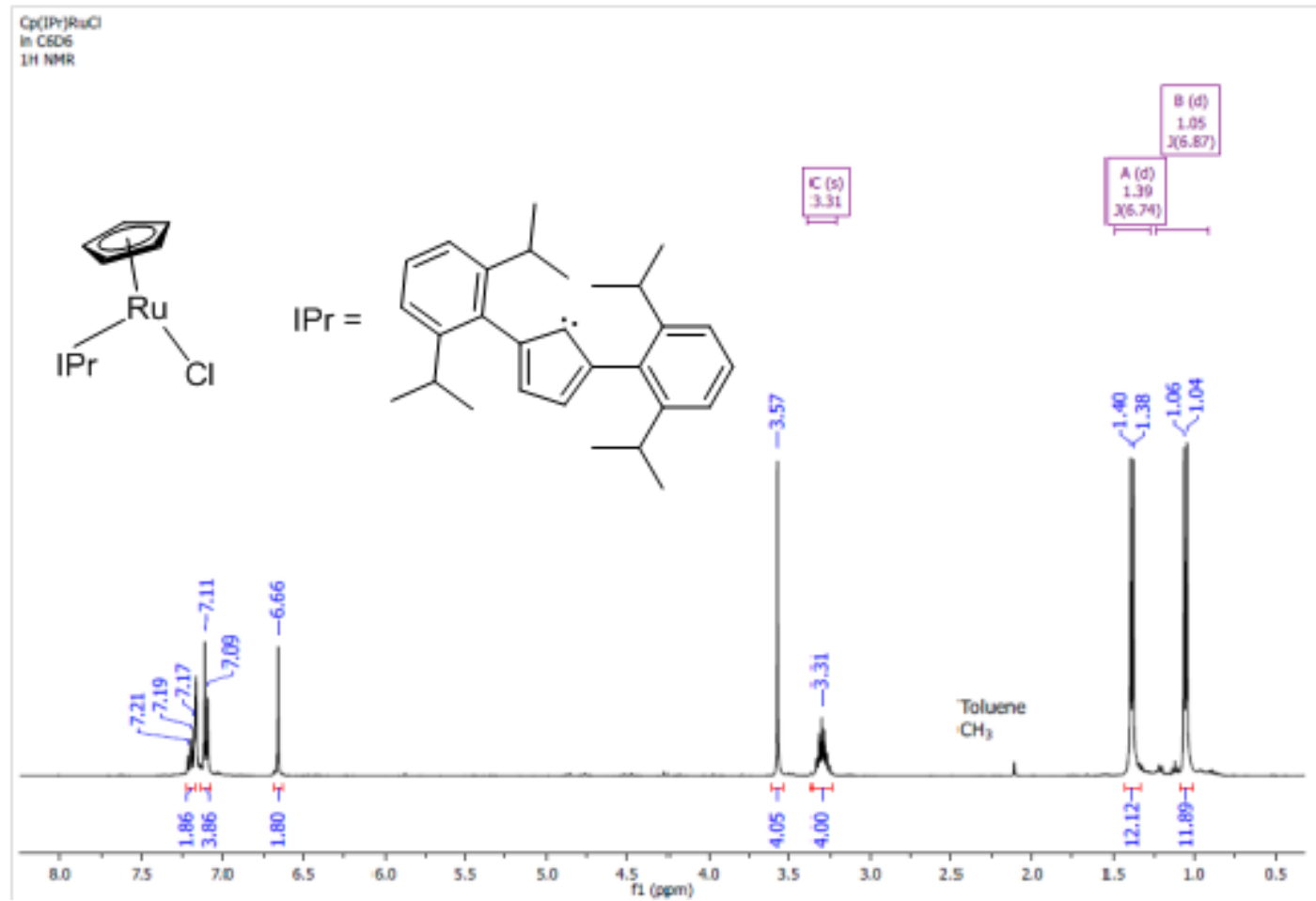

Figure S20. ${ }^{1} \mathrm{H}$ NMR $\left(400 \mathrm{MHz}, 22^{\circ} \mathrm{C}, \mathrm{C}_{6} \mathrm{D}_{6}\right)$ of $\mathrm{Cp}(\operatorname{IPr}) \mathrm{RuCl}(\mathbf{8})$.



Figure S21. ${ }^{13} \mathrm{C}\left\{{ }^{1} \mathrm{H}\right\}$ NMR spectrum $\left(100.6 \mathrm{MHz}, 22{ }^{\circ} \mathrm{C}, \mathrm{C}_{6} \mathrm{D}_{6}\right)$ of $\mathrm{Cp}(\mathrm{IPr}) \mathrm{RuCl}(\mathbf{8})$. 
$\left[\mathrm{Cp}(\mathrm{IPr}) \mathrm{Ru}\left(\mathrm{NCCH}_{3}\right)_{2}\right] \mathrm{BArF}(\mathbf{9})$

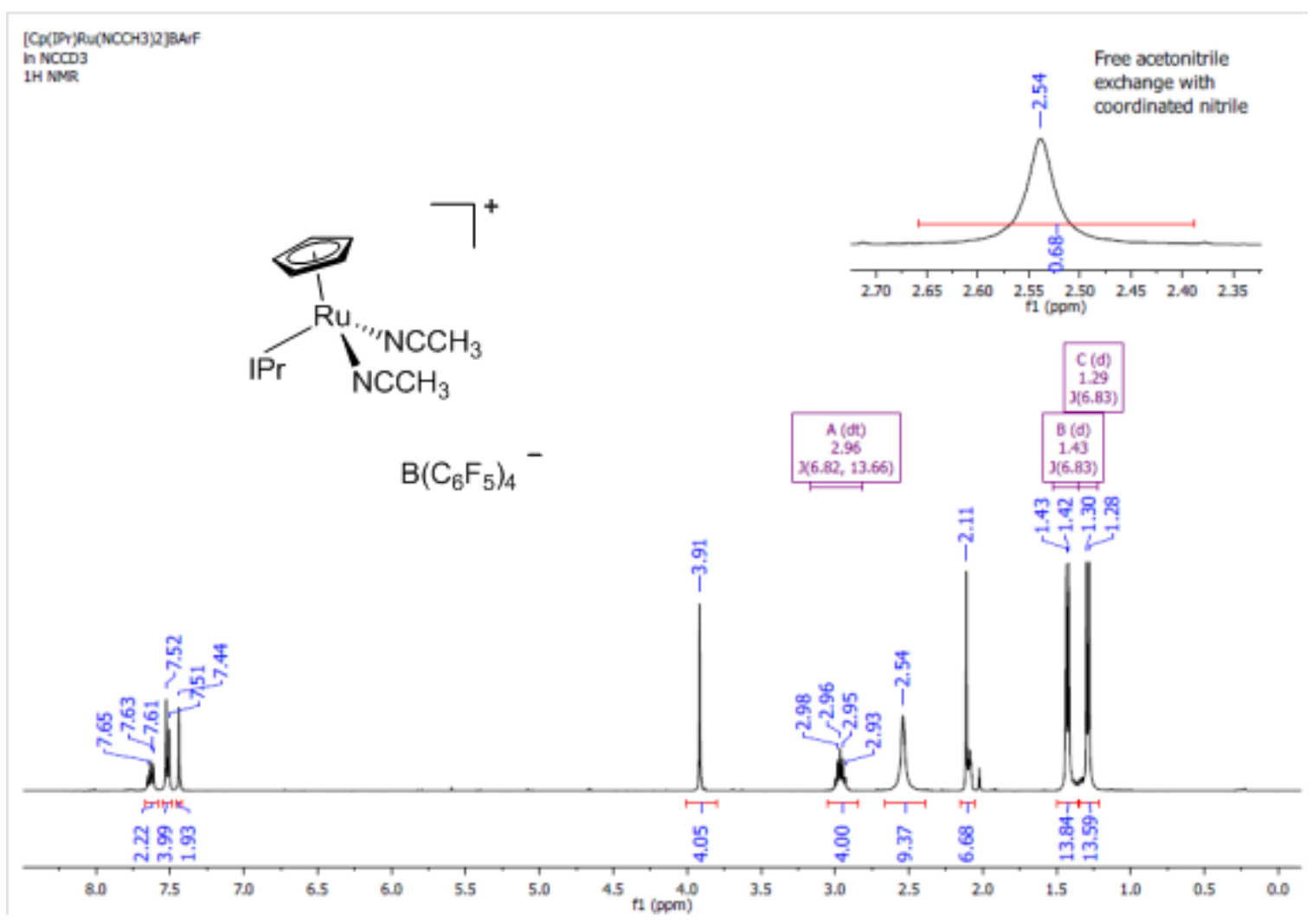

Figure S22. ${ }^{1} \mathrm{H}$ NMR $\left(600 \mathrm{MHz}, 22^{\circ} \mathrm{C}, \mathrm{NCCD}_{3}\right)$ of $\left[\mathrm{Cp}(\mathrm{IPr}) \mathrm{Ru}\left(\mathrm{NCCH}_{3}\right)_{2}\right] \mathrm{BArF}(9)$

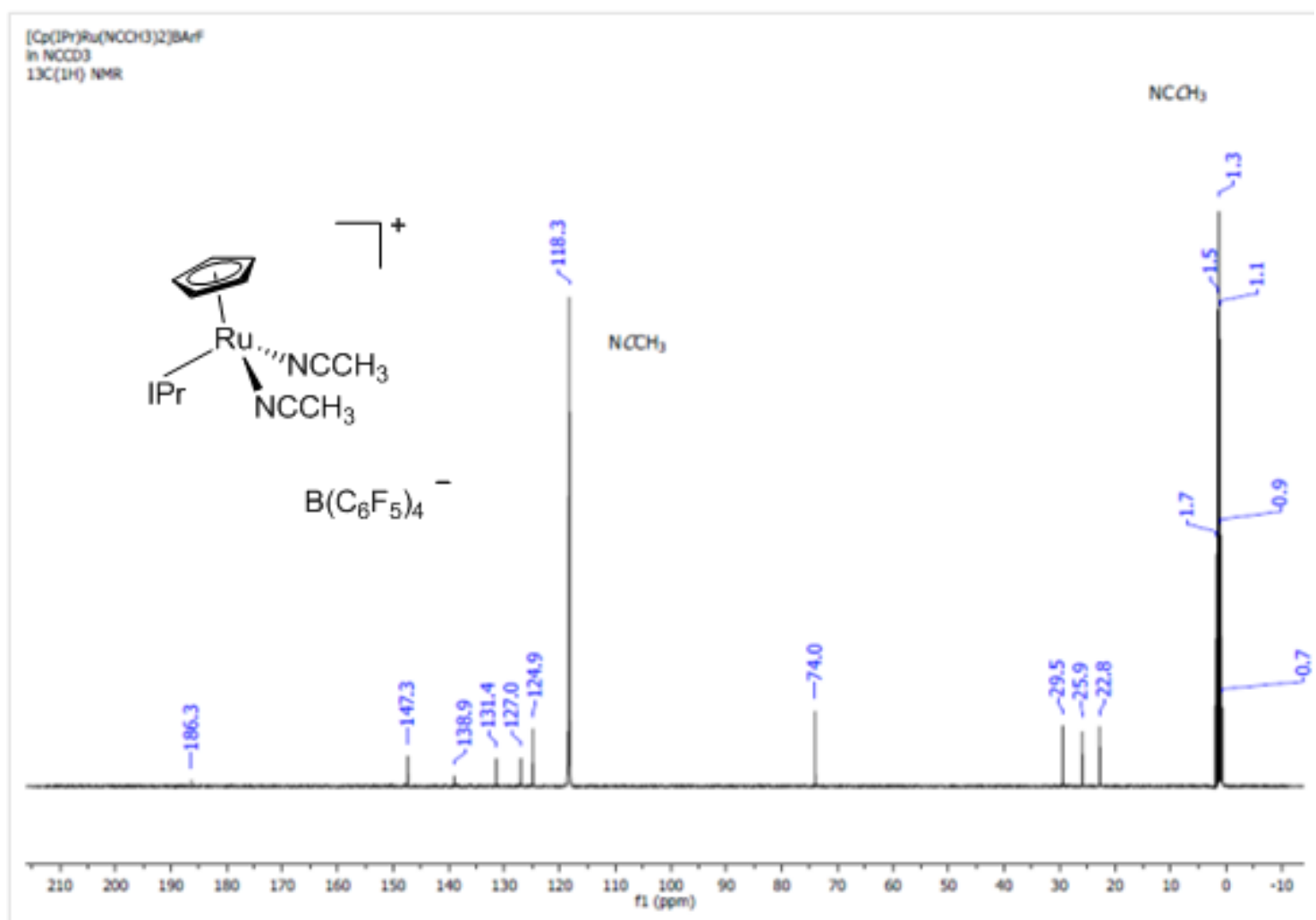

Figure S23. ${ }^{13} \mathrm{C}\left\{{ }^{1} \mathrm{H}\right\}$ NMR spectrum $\left(151 \mathrm{MHz}, 22^{\circ} \mathrm{C}, \mathrm{NCCD} \mathrm{D}_{3}\right)$ of $\left[\mathrm{Cp}(\mathrm{IPr}) \mathrm{Ru}\left(\mathrm{NCCH}_{3}\right)_{2}\right] \mathrm{BArF}(\mathbf{9})$ 

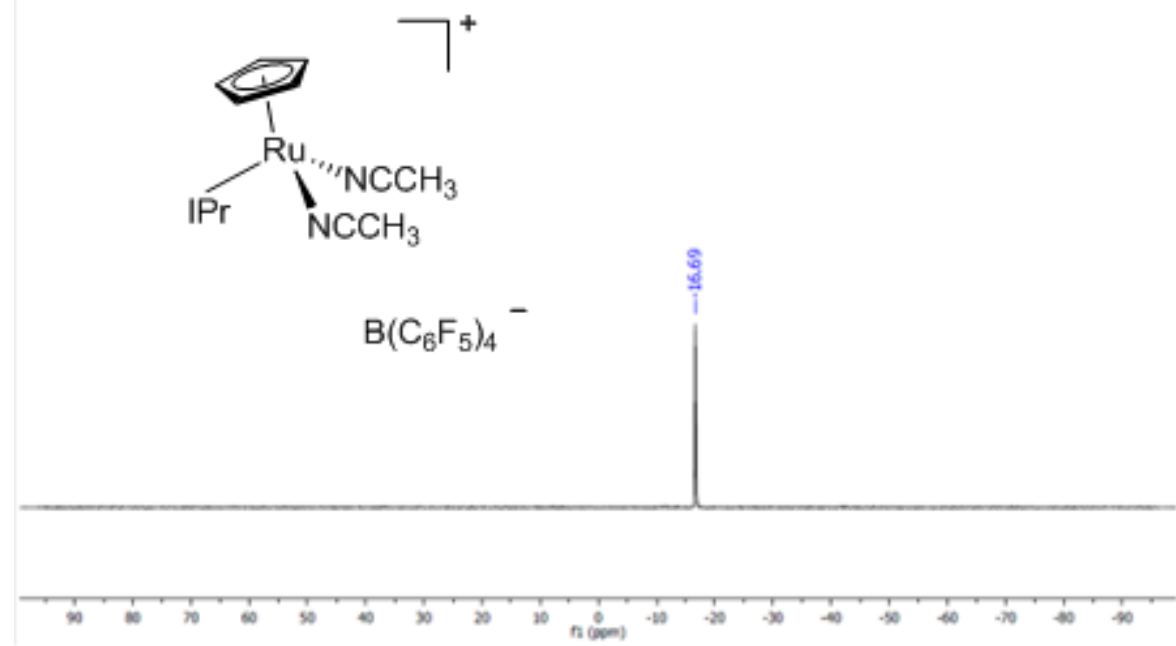

Figure S24. ${ }^{11} \mathrm{~B}\left\{{ }^{1} \mathrm{H}\right\}$ NMR spectrum $\left(151 \mathrm{MHz}, \mathrm{NCCD}_{3}\right)$ of $\left[\mathrm{Cp}(\mathrm{IPr}) \mathrm{Ru}\left(\mathrm{NCCH}_{3}\right)_{2}\right] \mathrm{B} \operatorname{ArF}(\mathbf{9})$

$[\mathrm{IPrH}]\left[\mathrm{CpRuCl}(\mathrm{H})\left(\mathrm{SiCl}_{3}\right)_{2}\right](\mathbf{1 0})$



Figure S25. ${ }^{1} \mathrm{H}$ NMR $\left(600 \mathrm{MHz}, 22^{\circ} \mathrm{C}, \mathrm{CD}_{2} \mathrm{Cl}_{2}\right)$ of $[\mathrm{IPrH}]\left[\mathrm{CpRuCl}(\mathrm{H})\left(\mathrm{SiCl}_{3}\right)_{2}\right](\mathbf{1 0})$ 


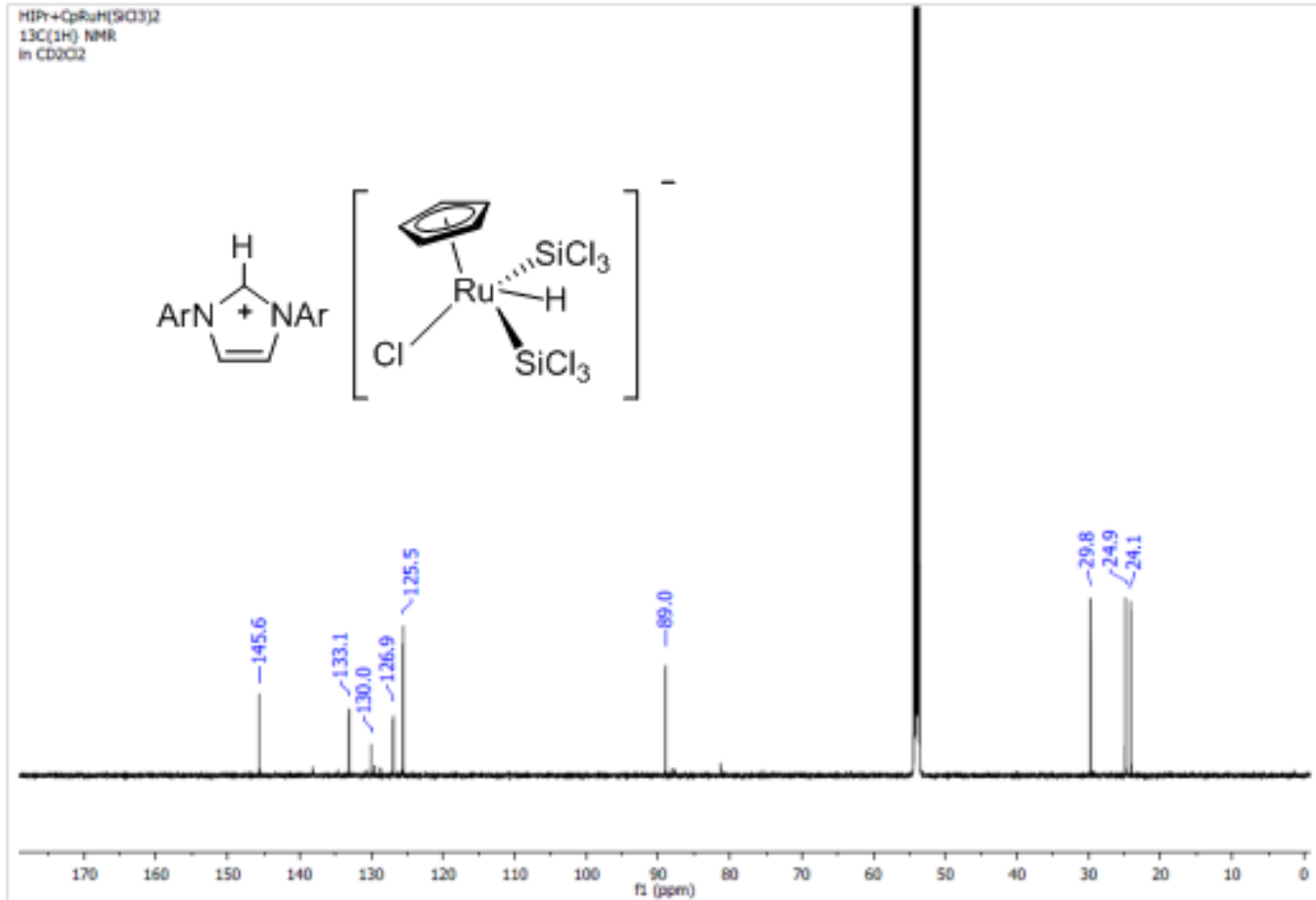

Figure S26. ${ }^{13} \mathrm{C}\left\{{ }^{1} \mathrm{H}\right\} \mathrm{NMR}\left(600 \mathrm{MHz}, 22^{\circ} \mathrm{C}, \mathrm{CD}_{2} \mathrm{Cl} 2\right)$ of $[\mathrm{IPrH}]\left[\mathrm{CpRuCl}(\mathrm{H})\left(\mathrm{SiCl}_{3}\right)_{2}\right](\mathbf{1 0})$

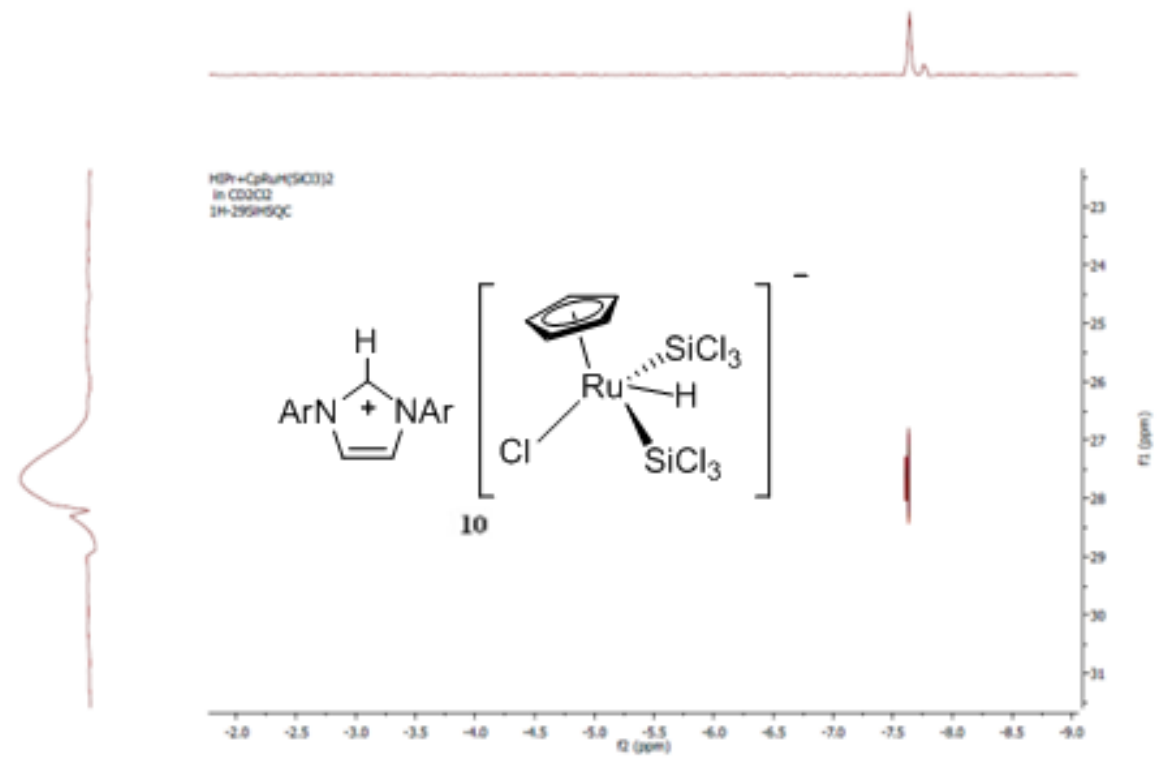

Figure S27. ${ }^{1} \mathrm{H}-{ }^{29} \mathrm{Si} \mathrm{HSQC} \mathrm{NMR}\left(119.2 \mathrm{MHz}, 22^{\circ} \mathrm{C}, \mathrm{CD}_{2} \mathrm{Cl}_{2}\right)$ of $[\mathrm{IPrH}]\left[\mathrm{CpRuCl}(\mathrm{H})\left(\mathrm{SiCl}_{3}\right)_{2}\right](\mathbf{1 0})$ 